\title{
Output Privacy in Data Mining
}

\author{
TING WANG \\ Georgia Institute of Technology \\ and \\ LING LIU \\ Georgia Institute of Technology
}

\begin{abstract}
Privacy has been identified as a vital requirement in designing and implementing data mining systems. In general, privacy preservation in data mining demands protecting both input and output privacy: the former refers to sanitizing the raw data itself before performing mining; while the latter refers to preventing the mining output (models or patterns) from malicious inference attacks. This paper presents a systematic study on the problem of protecting output privacy in data mining, and particularly, stream mining: (i) we highlight the importance of this problem by showing that even sufficient protection of input privacy does not guarantee that of output privacy; (ii) we present a general inferencing and disclosure model that exploits the intra-window and inter-window privacy breaches in stream mining output; (iii) we propose a light-weighted countermeasure that effectively eliminates these breaches without explicitly detecting them, while minimizing the loss of output accuracy; (iv) we further optimize the basic scheme by taking account of two types of semantic constraints, aiming at maximally preserving utility-related semantics while maintaining hard privacy guarantee; (v) finally, we conduct extensive experimental evaluation over both synthetic and real data to validate the efficacy of our approach.
\end{abstract}

Categories and Subject Descriptors: H.2.8 [Database Management]: Database Applicationsdata mining; H.2.7 [Database Management]: Database Administration-security, integrity, and protection

General Terms: Security, Algorithm, Experimentation

Additional Key Words and Phrases: Output privacy, stream mining, data perturbation

\section{INTRODUCTION}

Privacy of personal information has been arising as a vital requirement in designing and implementing data mining and management systems; individuals were usually unwilling to provide their personal information if they knew that the privacy of the data could be compromised. To this end, a plethora of work has been done on preserving input privacy for static data [Agrawal and Srikant 2000; Sweeney 2002; Evfimievski et al. 2002; Chen and Liu 2005; Machanavajjhala et al. 2006], which assumes untrusted data recipients and enforces privacy regulations by sanitizing the raw data before sending it to the recipients. The mining algorithms are performed over the sanitized data, and produce output (patterns or models) with accuracy comparable to, if not identical to that constructed over the raw data. This scenario is illustrated as the first four steps of the grand framework of privacy-preserving data mining in Fig. 1.

Nevertheless, in a strict sense, privacy preservation not only requires to prevent unauthorized access to raw data that leads to exposure of sensitive information, but also includes eliminating unwanted disclosure of sensitive patterns through inference attacks over mining output. By sensitive patterns, we refer to those 


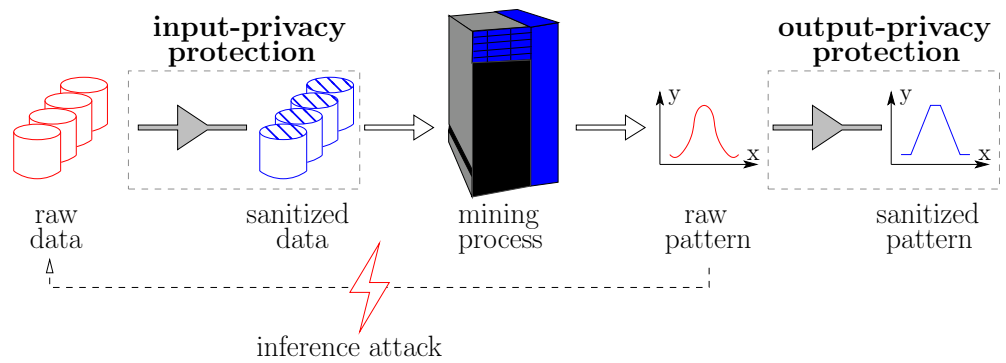

Fig. 1. Grand framework of privacy-preserving data mining.

properties possessed uniquely by a small number of individuals participating in the input data. At the first glance, it may seem sufficient to sanitize input data in order to address such threat; however, as will be revealed, even though the patterns (or models) are built over the sanitized data, the published mining output could still be leveraged to infer sensitive patterns. Intuitively, this can be explained by the fact that input-privacy protection techniques are designed to make the constructed models close to, if not identical to that built over the raw data, in order to guarantee the utility of the result. Such "no-outcome-change" property is considered as a key requirement of privacy-preserving data mining [Bu et al. 2007]. Given that the significant statistical information of the raw data is preserved, there exists the risk of disclosure of sensitive information. Therefore, the preservation of input privacy may not necessarily lead to that of output privacy, while it is necessary to introduce another unique layer of output-privacy protection into the framework, as shown in Fig. 1. A concrete example is given as follows.

Example 1.1. Consider a nursing-care records database that collects the observed symptoms of the patients in a hospital. By mining such database, one can discover valuable information regarding syndromes characterizing particular diseases. However, the released mining output can also be leveraged to uncover some combinations of symptoms that are so special that only rare people match them (we will show how to achieve this in the following sections), which qualifies as a severe threat to individuals' privacy.

Assume that Alice knows that Bob has certain symptoms $a, b$ but not $c(\bar{c})$, and by analyzing the mining output, she finds that only one person in the hospital matching the specific combination of $\{a, b, \bar{c}\}$, and only one having all $\{a, b, \bar{c}, d\}$. She can safely conclude that the victim is Bob, who also suffers the symptom d. Further more, by studying other medical databases, she may learn that the combination of $\{a, b, d\}$ is linked to a rare disease with fairly high chance.

The output-privacy issue is more complicated in stream mining, wherein the mining output usually needs to be published in a continuous and in-time manner. Not only a single-time release may contain privacy breaches, but also multiple releases can potentially be exploited in combination, given the overlap of the corresponding input data. Consider the sliding window model [Babcock et al. 2002] as an example, arguably the most popular stream processing model, where queries are not evaluated over the entire history of the stream, but rather over a sliding window of the 
most recent data from the stream. The window may be defined over data items or timestamps, i.e., item-based or time-based window, respectively. Besides the leakage in the output of a single window (intra-window breach), the output of multiple overlapping windows could also be combined to infer sensitive information (interwindow breach), even each window itself contains no breach per se. Moreover, the characteristics of the stream typically evolve over time, which precludes the feasibility of global data analysis-based techniques, due to the strict processing time and memory limitations. Hence, one needs to consider addressing output-privacy vulnerabilities in stream mining systems as a unique problem.

Surprisingly, in contrast of the wealth of work on protecting input privacy, output privacy has received fairly limited attention so far in both stream data mining and privacy-preserving data mining in general. This work, to our best knowledge, represents the most systematic study to date of output-privacy vulnerabilities in the context of stream data mining.

\subsection{State of the Art}

The first naturally arising question might be: is it sufficient to apply input-privacy protection techniques to address output vulnerabilities? Unfortunately, most existing techniques fail to satisfy the requirement of countering inference attacks over mining output: they differ from one to another in terms of concrete mechanisms to provide attack-resilient protection while minimizing utility loss of mining output incurred by sanitization; however, the adversarial attacks over input data (raw records) is significantly different from that over mining output (patterns or models), which renders these techniques inapplicable for our purpose.

As a concrete case, in Example 1.1, one conceivable solution to controlling the inference is to block or perturb those sensitive records, e.g., the one corresponding to Bob, in the mining process; however, such record-level perturbation suffers from a number of drawbacks. First, the utility of mining output is not guaranteed. Since the perturbation directly affects the mining output, it is usually difficult to guarantee both that the valuable knowledge (the intended result) is preserved and that the sensitive patterns are disguised. Among these, one significant issue is that it may result in a large amount of false knowledge. For instance, in Example 1.1, if the dataset is prepared for frequent pattern mining, blocking or perturbing sensitive records may make frequent patterns become non-frequent, or vice versa; if the dataset is prepared for learning classification tree, modifying sensitive records may result in significant deviation of the cut points, which are critical for decision making. Second, unlike the scenarios considered in some existing work (e.g., [Wang et al. 2007]), in real applications, the sensitive patterns may not be predefined or directly observable; rather, sophisticated analysis over the entire dataset is typically necessary to detect the potential privacy leakage of mining output. For example, as we will show in Section 3, in the case of frequent pattern mining that involves a lattice structure among the support of itemsets, the number of potential breaches needed to be checked is exponential in terms of the number of items. The situation is even more complicated for the case of stream mining case wherein multiple windows can be exploited together for inference. Such complexity imposes efficiency issues for record-level perturbation. Third, in a broad range of computation-intensive applications, e.g., neural network-based models, the mining output is typically not 
directly observable; thus the effect of applying record-level perturbation cannot be evaluated without running the mining process. In all theses cases, it is difficult to perform record-level perturbation to protect sensitive patterns.

Meanwhile, one might draw a comparison between our work and the disclosure control techniques in statistical and census databases. Both concern about providing statistical information without compromising sensitive information regarding individuals; however, they also exhibit significant distinctions. First, the queries of statistical databases typically involve only simple statistics, e.g., MIN, MAX, AVG, etc., while the output (patterns or models) of data mining applications usually feature much more complex structures, leading to more complicated requirements for output utility. Second, compared with that in statistical databases, the outputprivacy protection in data mining faces much stricter constraints over processing time and space, which is especially true for the case of stream mining.

\subsection{Overview of Our Solution}

A straightforward yet inefficient solution to preserving output privacy is to detect and eliminate all potential breaches, i.e., the detecting-then-removing paradigm as typically adopted by inference control in statistical databases. However, the detection of breaches usually requires computation-intensive analysis of the entire dataset, which is negative in tone [Chin and Ozsoyoglu 1981] for stream mining systems. Further, even at such high cost, the concrete operations of removing the identified breaches, e.g., suppression and addition [Atzori et al. 2008], tend to result in considerable decrease in the utility of mining output.

Instead, we propose a novel proactive model to counter inference attacks over output. Analogous to sanitizing raw data from leaking sensitive information, we introduce the concept of "sanitized pattern", arguing that by intelligently modifying the "raw patterns" produced by mining process, one is able to significantly reduce the threat of malicious inference, while maximally preserving the utility of raw patterns. This scenario is shown as the last step in Fig. 1.

In contrary to record-level perturbation, pattern-level perturbation demonstrates advantages in both protecting sensitive patterns and preserving output utility. First, the utility of mining output is guaranteed, e.g., it is feasible to precisely control the amount of false knowledge. For instance, in Example 1.1, all the valuable frequent patterns regarding symptom-disease relationships can be preserved, while no false frequent patterns are introduced. Also, as we will show in Section 5 and 6 , in the case of frequent pattern mining, not only the accuracy of each frequent itemset can be controlled, but also their semantic relationships can be preserved to the maximum extent, which is hard to achieve with record-level perturbation. Second, it is possible to devise effective yet efficient pattern-level perturbation schemes that can be performed either online or offline, without affecting the efficiency of (stream) mining process. Finally, since the target of perturbation, the mining output, is directly observable to the perturbation process, it is possible to analytically gauge the perturbation schemes.

Specifically, we present BuTTERFLY*, a light-weighted countermeasure against malicious inference over mining output. It possesses a series of desirable features that make it suitable for (stream) mining applications: (i) it needs no explicit detection of (either intra-window or inter-window) privacy breaches; (ii) it requires

ACM Transactions on Database Systems, Vol. , No. , 20 
no reference to previous output when publishing the current result; (iii) it provides flexible control over the balance of multiple utility metrics and privacy guarantee.

Following a two-phase paradigm, BuTTERFLY* achieves attack-resilient protection and output-utility preservation simultaneously: in the first phase, it counters malicious inference by amplifying the uncertainty of sensitive patterns, at the cost of trivial accuracy loss of individual patterns; in the second phase, while guaranteeing the required privacy, it maximally optimizes the output utility by taking account of several model-specific semantic constraints.

Our contributions can be summarized as follows: (i) we articulate the problem and the importance of preserving output privacy in (stream) data mining; (ii) we expose a general inference attack model that exploits the privacy breaches existing in current (stream) mining systems; (iii) we propose a two-phase framework that effectively addresses attacks over (stream) mining output; (iv) we provide both theoretical analysis and experimental evaluation to validate our approach in terms of privacy guarantee, output utility, and execution efficiency.

\subsection{Paper Roadmap}

We begin in Section 2 with introducing the preliminaries of frequent pattern mining over data streams, exemplifying with which, we formalize the problem of addressing output-privacy vulnerabilities in (stream) data mining. In Section 3, after introducing a set of basic inferencing techniques, we present two general attack models that exploit intra-window and inter-window privacy breaches in stream mining output, respectively. Section 4 outlines the motivation and design objectives of ButTERFLY*, followed by Section 5 and 6 detailing the two phases of ButTERFLY* and discussing the implicit trade-offs among privacy guarantee and multiple utility metrics. Section 7 examines the impact of perturbation distribution over the quality of privacy protection and utility preservation. An empirical evaluation of the analytical models and the efficacy of Butterfly* is presented in Section 8. Finally, section 9 surveys relevant literature, and the paper is concluded in Section 10.

\section{PROBLEM FORMALIZATION}

To expose the output-privacy vulnerabilities in existing mining systems, we exemplify with the case of frequent pattern mining over data streams. We first introduce the preliminary concepts of frequent pattern mining and pattern categorization, and then formalize the problem of protecting output privacy in such mining task.

\subsection{Frequent Pattern Mining}

Consider a finite set of items $\mathcal{I}=\left\{i_{1}, i_{2}, \ldots, i_{M}\right\}$. An itemset $I$ is a subset of $\mathcal{I}$, i.e., $I \subseteq \mathcal{I}$. A database $\mathcal{D}$ consists of a set of records, each corresponds to a nonempty itemset. The support of an itemset $I$ with respect to $\mathcal{D}$, denoted by $T_{\mathcal{D}}(I)$, is defined as the number of records containing $I$ as a subset in $\mathcal{D}$. Frequent pattern mining aims at finding all itemsets with support exceeding a predefined threshold $C$, called minimum support.

A data stream $\mathcal{S}$ is modeled as a sequence of records, $\left(r_{1}, r_{2}, \ldots, r_{N}\right)$, where $N$ is the current size of $\mathcal{S}$, and grows as time goes by. The sliding window model is introduced to deal with the potential of $N$ going to infinity. Concretely, at each $N$, one considers only the window of most recent $H$ records, $\left(r_{N-H+1}, \ldots, r_{N}\right)$, 


\begin{tabular}{|c|c|c|c|c|c|c|c|c|c|c|c|c|c|}
\hline \multirow[b]{2}{*}{$\ldots$} & \multirow[b]{2}{*}{$r_{1}$} & \multirow[b]{2}{*}{$r_{2}$} & \multirow[b]{2}{*}{$r_{3}$} & \multicolumn{9}{|c|}{$\mathrm{S}(12,8)$} & \multirow[b]{2}{*}{$\ldots$} \\
\hline & & & & $r_{4}$ & $r_{5}$ & $r_{6}$ & $r_{7}$ & $r_{8}$ & $r_{9}$ & $r_{10}$ & $r_{11}$ & $r_{12}$ & \\
\hline \multirow{2}{*}{$\cdots$} & $\begin{array}{l}a \\
b\end{array}$ & a & $\begin{array}{l}a \\
b\end{array}$ & $\begin{array}{l}a \\
b\end{array}$ & $\mathrm{a}$ & $\begin{array}{l}a \\
b\end{array}$ & $\begin{array}{l}a \\
b\end{array}$ & b & $\begin{array}{l}\mathrm{a} \\
\mathrm{b}\end{array}$ & a & b & & \multirow{2}{*}{$\cdots$} \\
\hline & $\begin{array}{l}c \\
d\end{array}$ & C & $\mathrm{d}$ & $\begin{array}{l}c \\
d\end{array}$ & $C$ & C & $C$ & $\begin{array}{l}c \\
d\end{array}$ & $\begin{array}{l}c \\
d\end{array}$ & C & $\begin{array}{l}c \\
d\end{array}$ & $\begin{array}{l}c \\
d\end{array}$ & \\
\hline
\end{tabular}

Fig. 2. Data stream and sliding window model.

denoted by $\mathcal{S}(N, H)$, where $H$ is the window size. The problem is therefore to find all the frequent itemsets in each window.

Example 2.1. Consider a data stream with current size $N=12$, window size $H$ $=8$, as shown in Fig. 2, where $a \sim d$ and $r_{1} \sim r_{12}$ represent the set of items and records, respectively. Assuming minimum support $C=4$, then within window $\mathcal{S}(11,8),\{c, b c, a c, a b c\}$ is a subset of frequent itemsets.

One can further generalize the concept of itemset by introducing the negation of an item. Let $\bar{i}$ denote the negation of the item $i$. A record is said to contain $\bar{i}$ if it does not contain $i$. Following, we will use the term pattern to denote a set of items or negation of items, e.g., $a \overline{b c}$. We use $\bar{I}$ to denote the negation of an itmeset $I$, i.e., $\bar{I}=\{\bar{i} \mid i \in I\}$.

Analogously, we say that a record $r$ satisfies a pattern $P$ if it contains all the items and negations of items in $P$ and the support of $P$ with respect to database $\mathcal{D}$ is defined as the number of records containing $P$ in $\mathcal{D}$.

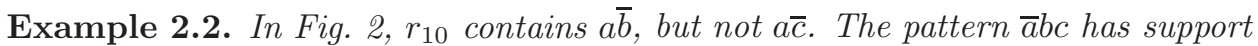
2 with respect to $\mathcal{S}(12,8)$, because only records $r_{8}$ and $r_{11}$ match it.

\subsection{Pattern Categorization}

Loosely speaking, output privacy refers to the requirement that the output of mining process does not disclose any sensitive information regarding individuals participating in input data.

In the context of frequent pattern mining, such sensitive information can be instantiated as patterns with extremely low support, which correspond to properties uniquely possessed by few records (individuals), as shown in Example 1.1. We capture this intuition by introducing a threshold $K(K \ll C)$, called vulnerable support, and consider patterns with (non-zero) support below $K$ as vulnerable patterns. We can then establish the following classification system.

Definition 2.3. (Pattern Categorization) Given a database $\mathcal{D}$, let $\mathcal{P}$ be the set of patterns appearing in $\mathcal{D}$, then all $P \in \mathcal{P}$ can be classified into three disjoint classes, for the given threshold $K$ and $C$.

$$
\begin{cases}\text { Frequent Pattern }(\mathrm{FP}): & \mathcal{P}_{f}=\left\{P \mid T_{\mathcal{D}}(P) \geq C\right\} \\ \text { Hard Vulnerable Pattern (HVP) }: & \mathcal{P}_{h v}=\left\{P \mid 0<T_{\mathcal{D}}(P) \leq K\right\} \\ \text { Soft Vulnerable Pattern (SVP) : } & \mathcal{P}_{s v}=\left\{P \mid K<T_{\mathcal{D}}(P)<C\right\}\end{cases}
$$

ACM Transactions on Database Systems, Vol. , No. , 20. 
Intuitively, frequent pattern $\left(\mathcal{P}_{f}\right)$ is the set of patterns with support above minimum support $C$; they expose the significant statistics of the underlying data, and are often the candidate in the mining process. Actually the frequent itemsets found by frequent pattern mining are a subset of $\mathcal{P}_{f}$. Hard vulnerable pattern $\left(\mathcal{P}_{h v}\right)$ is the set of patterns with support below vulnerable support $K$; they represent the properties possessed by only few individual, so it is unacceptable that they are disclosed or inferred from mining output. Finally, soft vulnerable pattern $\left(\mathcal{P}_{s v}\right)$ neither demonstrates the statistical significance, nor violates the privacy of individual records; such patterns are not contained in mining output, but it is usually tolerable that they are learned from the output.

Example 2.4. As shown in Fig. 2, given $K=1$ and $C=4$, ac and bc are both $\mathcal{P}_{f}$, and $\overline{a b} c$ is $\mathcal{P}_{h v}$ with respect to $\mathcal{S}(12,8)$, while bcd is $\mathcal{P}_{\text {sv }}$ since its support lies between $K$ and $C$.

\subsection{Problem Definition}

We are now ready to formalizing the problem of preserving output privacy in the context of frequent pattern mining over streams: For each sliding window $\mathcal{S}(N, H)$, output-privacy preservation prevents the disclosure or inference of any hard vulnerable patterns with respect to $\mathcal{S}(N, H)$ from the mining output.

It may seem at the first glance that no breach exists at all in frequent pattern mining, if it only outputs frequent itemsets (recall $C \gg K$ ); however, as will be revealed shortly, from the released frequent patterns and their associated support, the adversary may still be able to infer certain hard vulnerable patterns, as shown in the next example (with detailed discussion in Section 3).

Example 2.5. Recall Example 2.4. Given the support of $\{c, a c, b c, a b c\}$, based on the inclusion-exclusion principle [O'Connor 1993], $T(\overline{a b} c)=T(c)-T(a c)-T(b c)+$ $T(a b c)$, one is able to infer the support of $\overline{a b} c$, which is $\mathcal{P}_{h v}$ in $\mathcal{S}(12,8)$.

\section{ATTACK OVER MINING OUTPUT}

In this section, we reveal the privacy breaches existing in current (stream) mining systems, and present a general attack model that can exploit these breaches.

\subsection{Attack Model}

For simplicity of presentation, we will use the following notations: given two itemsets $I$ and $J, I \oplus J$ denotes their union, $I \odot J$ their intersection, $J \ominus I$ the set difference of $J$ and $I$, and $|I|$ the size of $I$. The notations used in the rest of the paper are listed in Table I.

As a special case of multi-attribute aggregation, computing the support of $I$ $(I \subseteq J)$ can be considered as generalization of $J$ over all the attributes of $J \ominus I$; therefore, one can apply the standard tool of multi-attribute aggregation, a lattice structure, based on which we construct the attack model.

Lattice Structure. Consider two itemsets $I, J$ that satisfy $I \subset J$. All the itemsets $\mathcal{X}_{I}^{J}=\{X \mid I \subseteq X \subseteq J\}$ form a lattice structure: Each node corresponds to an itemset $X$, and each edge represents the generalization relationship between two 


\begin{tabular}{c|l}
\hline notation & description \\
\hline \hline $\mathcal{S}(N, H)$ & stream window of $\left(r_{N-H+1} \sim r_{N}\right)$ \\
$T_{\mathcal{D}}(X)$ & support of $X$ in database $\mathcal{D}$ \\
$K$ & vulnerable support \\
$C$ & minimum support \\
$\mathcal{X}_{I}^{J}$ & set of itemsets $\{X \mid I \subseteq X \subseteq J\}$ \\
$W_{p}$ & previous window \\
$W_{c}$ & current window \\
$\Delta_{X}^{+}$ & number of inserted records containing $X$ \\
$\Delta_{X}^{-}$ & number of deleted records containing $X$ \\
\hline
\end{tabular}

Table I. Symbols and notations.

nodes $X_{s}$ and $X_{t}$ such that $X_{s} \subset X_{t}$ and $\left|X_{t} \ominus X_{s}\right|=1$. Namely, $X_{s}$ is the generalization of $X_{t}$ over the item $X_{t} \ominus X_{s}$.

Example 3.1. A lattice structure is shown in Fig. 3, where $I=c, J=a b c$, and $J \ominus I=a b$.

For simplicity, in what follows, we use $\mathcal{X}_{I}^{J}$ to represent both the set of itemsets and their corresponding lattice structure. Next, we introduce the basis of our inferencing model, namely, deriving pattern support and estimating itemset support. These two techniques have been introduced in [Atzori et al. 2008] and [Calders and Goethals 2002], respectively, with usage or purpose different from ours. In [Atzori et al. 2008], deriving pattern support is considered as the sole attack model to uncover sensitive patterns; in [Calders and Goethals 2002], estimating itemset support is used to mine non-derivable patterns, and thus saving the storage of patterns. The novelty of our work, however, lies in constructing a general inferencing model that exploits the privacy breaches existing in single or multiple releases of mining output, with these two primitives as building blocks.

Deriving Pattern Support. Consider two itemsets $I \subset J$, if the support of all the lattice nodes of $\mathcal{X}_{I}^{J}$ is accessible, one is able to derive the support of pattern $P$, $P=I \oplus(\overline{J \ominus I})$, according to the inclusion-exclusion principle [O'Connor 1993]:

$$
T(I \oplus(\overline{J \ominus I}))=\sum_{I \subseteq X \subseteq J}(-1)^{|X \ominus I|} T(X)
$$

Example 3.2. Recall the example illustrated in Fig. 3. Given the support of the lattice nodes of $\mathcal{X}_{c}^{a b c}$ in $\mathcal{S}(12,8)$, the support of pattern $P=\overline{a b} c$ is derived as: $T_{\mathcal{S}(12,8)}(\overline{a b} c)=T_{\mathcal{S}(12,8)}(c)-T_{\mathcal{S}(12,8)}(a c)-T_{\mathcal{S}(12,8)}(b c)+T_{\mathcal{S}(12,8)}(a b c)=1$.

Essentially, the adversary can use this technique to infer vulnerable patterns with respect to one specific window from mining output.

Estimating Itemset Support. For the support of any itemset is non-negative, according to the inclusion-exclusion principle, if the support of the itemsets $\{X \mid I \subseteq$ $X \subset J\}$ is available, one is able to bound the support of $J$ as follows:

$$
\begin{cases}T(J) \leq \sum_{I \subseteq X \subset J}(-1)^{|J \ominus X|+1} T(X) & |J \ominus I| \text { is odd } \\ T(J) \geq \sum_{I \subseteq X \subset J}(-1)^{|J \ominus X|+1} T(X) & |J \ominus I| \text { is even }\end{cases}
$$



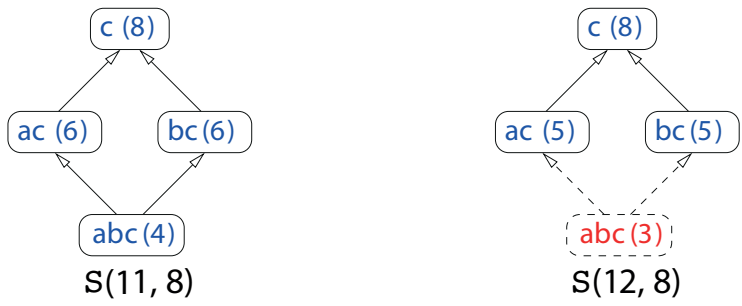

Fig. 3. Privacy breaches in stream mining output.

Example 3.3. Given the support of $c$, ac, and bc in $\mathcal{S}(12,8)$, one is able to establish the lower and upper bounds of $T_{\mathcal{S}(12,8)}(a b c)$ as: $\leq T_{\mathcal{S}(12,8)}(a c)=5, \leq T_{\mathcal{S}(12,8)}(b c)=$ $5, \geq T_{\mathcal{S}(12,8)}(a c)+T_{\mathcal{S}(12,8)}(b c)-T_{\mathcal{S}(12,8)}(c)=2$.

When the bounds are tight, i.e., the lower bound meets the upper bound, one can exactly determine the actual support. In our context, the adversary can leverage this technique to uncover the information regarding certain unpublished itemsets.

\subsection{Intra-Window Inference}

In stream mining systems without output-privacy protection, the released frequent itemsets over one specific window may contain intra-window breaches, which can be exploited via the technique of deriving or estimating pattern support.

Example 3.4. As shown in Example 3.2, $\overline{a b} c$ is $\mathcal{P}_{h v}$ with respect to $\mathcal{S}(12,8)$ if $K=$ 1 ; however, one can easily derive its support if the support values of $c, a c, b c, a b c$ are known.

Formally, if $J$ is a frequent itemset, then according to the Apriori rule [Agrawal and Srikant 1994], all $X \subseteq J$ must be frequent, which are supposed to be reported with their support. Therefore, the information is complete to compute the support of pattern $P=I \oplus(\overline{J \ominus I})$ for all $I \subset J$. This also implies that the number of breaches needed to be checked is potentially exponential in terms of the number of items.

Even if the support of $J$ is unavailable, i.e, the lattice of $\mathcal{X}_{I}^{J}$ is incomplete to infer $P=I \oplus(\overline{J \ominus I})$, one can first apply the technique of estimating itemset support to complete some missing "mosaics", then derive the support of vulnerable patterns. Possibly the itemsets under estimation themselves may be vulnerable. Following, we assume that estimating itemset support is performed as a preprocessing step of the attack.

\subsection{Inter-Window Inference}

The intra-window inference attack is only a part of the story. In stream mining, privacy breaches may also exist in the output of overlapping windows. Intuitively, the output of a previous window can be leveraged to infer the vulnerable patterns within the current window, and vice versa, even though no vulnerable patterns can be inferred from the output of each window per se.

Example 3.5. Consider two windows $W_{p}=\mathcal{S}(11,8)$ and $W_{c}=\mathcal{S}(12,8)$ as shown in Fig. 2, with frequent itemsets summarized in Fig. 3. Assume $C=4$ and $K=$ 
1. In window $W_{p}$, no $\mathcal{P}_{h v}$ exists; while in $W_{c}$, abc is unaccessible (shown as dashed box). From the available information of $W_{c}$, the best guess about abc is [2,5], as discussed in Example 3.3. Clearly, this bound is not tight enough to infer that $\overline{a b} c$ is $\mathcal{P}_{h v}$. Both windows are thus immune to intra-window inference.

However, if one is able to derive that the support of abc decreases by 1 between $W_{p}$ and $W_{c}$, then based on the information released in $W_{p}$, which is $T_{W_{p}}(a b c)=4$, the exact value of abc in $W_{c}$ can be inferred, and $\overline{a b} c$ is uncovered.

The main idea of inter-window inference is to exactly estimate the transition of the support of certain itemsets between the previous and current windows. We below discuss how to achieve accurate estimation of such transition over two consecutive windows.

Without loss of generality, consider two overlapping windows $W_{p}=\mathcal{S}(N-L, H)$ and $W_{c}=\mathcal{S}(N, H)(L<H)$, i.e., $W_{c}$ is lagging $W_{p}$ by $L$ records (in the example above, $N=12, H=8$ and $L=1)$. Assume that the adversary attempts to derive the support of pattern $P=I \oplus(\overline{J \ominus I})$ in $W_{c}$. Let $\mathcal{X}_{p}$ and $\mathcal{X}_{c}$ be the subsets of $\mathcal{X}_{I}^{J}$ that are released or estimated from the output of $W_{p}$ and $W_{c}$, respectively. We assume that $\mathcal{X}_{p} \oplus \mathcal{X}_{c}=\mathcal{X}_{I}^{J}\left(\mathcal{X}_{I}^{J} \ominus \mathcal{X}_{c}=\mathcal{X}_{p} \ominus \mathcal{X}_{c}\right)$, i.e., the missing part in $\mathcal{X}_{c}$ can be obtained in $\mathcal{X}_{p}$. In Fig. $3, \mathcal{X}_{p}=\{c, a c, b c, a b c\}$, while $\mathcal{X}_{c}=\{c, a c, b c\}$.

For itemset $X$, let $\Delta_{X}^{+}$and $\Delta_{X}^{-}$be the number of records containing $X$ in the windows $\mathcal{S}(N, L)$ and $\mathcal{S}(N-H, L)$, respectively. Thus, the support change of $X$ over $W_{p}$ and $W_{c}$ can be modeled as inserting $\Delta_{X}^{+}$records and deleting $\Delta_{X}^{-}$ones, i.e., $T_{W_{c}}(X)=T_{W_{p}}(X)+\Delta_{X}^{+}-\Delta_{X}^{-}$.

Example 3.6. Recall our running example, with $N=12, H=8$, and $L=1$. $\mathcal{S}(N, L)$ corresponds to the record $r_{11}$ while $\mathcal{S}(N-H, L)$ refers to the record $r_{4}$. Clearly, $r_{4}$ contains ac, while $r_{11}$ does not; therefore, $T_{\mathcal{S}(12,8)}(a c)=T_{\mathcal{S}(11,8)}(a c)+$ $\Delta_{a c}^{+}-\Delta_{a c}^{-}=5$.

The adversary is interested in estimating $T_{W_{c}}\left(X^{*}\right)$ for $X^{*} \in \mathcal{X}_{p} \ominus \mathcal{X}_{c}$. The bound (min, $\max$ ) of $T_{W_{c}}\left(X^{*}\right)$ can be obtained by solving the following integer programming problem:

$$
\max (\min ) \quad T_{W_{p}}\left(X^{*}\right)+\Delta_{X^{*}}^{+}-\Delta_{X^{*}}^{-}
$$

satisfying the constraints:

$$
\begin{array}{ll}
\mathrm{R}_{1}: 0 \leq \Delta_{X}^{+}, \Delta_{X}^{-} \leq L & \\
\mathrm{R}_{2}: \Delta_{X}^{+}-\Delta_{X}^{-}=T_{W_{c}}(X)-T_{W_{p}}(X) \quad X \in \mathcal{X}_{p} \odot \mathcal{X}_{c} \\
\mathrm{R}_{3}: \Delta_{X}^{+}\left(\Delta_{X}^{-}\right) \leq \sum_{I \subseteq Y \subset X}(-1)^{|X \ominus Y|+1} \Delta_{Y}^{+}\left(\Delta_{Y}^{-}\right) & |X \ominus I| \text { is odd } \\
\mathrm{R}_{4}: \Delta_{X}^{+}\left(\Delta_{X}^{-}\right) \geq \sum_{I \subseteq Y \subset X}(-1)^{|X \ominus Y|+1} \Delta_{Y}^{+}\left(\Delta_{Y}^{-}\right) & |X \ominus I| \text { is even }
\end{array}
$$

Here, $\mathrm{R}_{1}$ stems from that $W_{p}$ differs from $W_{c}$ by $L$ records. When transiting from $W_{p}$ to $W_{c}$, the records containing $X$ that are deleted or added cannot exceed $L$. $\mathrm{R}_{2}$ amounts to saying that the support change $\left(\Delta_{X}^{+}-\Delta_{X}^{-}\right)$for those itemsets $X \in \mathcal{X}_{c} \odot \mathcal{X}_{p}$ is known. $\mathrm{R}_{3}$ and $\mathrm{R}_{4}$ are the application of estimating itemset support for itemsets in windows $\mathcal{S}(N, L)$ and $\mathcal{S}(N-H, L)$.

Sketchily, the inference process runs as follows: starting from the change of $X \in$ $\mathcal{X}_{p} \odot \mathcal{X}_{c}\left(\mathrm{R}_{2}\right)$, by using rules $\mathrm{R}_{1}, \mathrm{R}_{3}$, and $\mathrm{R}_{4}$, one attempts to estimate $\Delta_{X}^{+}\left(\Delta_{X}^{-}\right)$for $X \in \mathcal{X}_{p} \ominus \mathcal{X}_{c}$. It is noted that when the interval $L$ between $W_{p}$ and $W_{c}$ is small enough, the estimation can be fairly tight.

ACM Transactions on Database Systems, Vol. , No. , 20 
Example 3.7. Consider our running example, $L=1$, and $\mathcal{X}_{p} \odot \mathcal{X}_{c}=\{c, a c, b c\}$. One can first observe the following facts based on $\mathrm{R}_{1}$ and $\mathrm{R}_{2}$ :

$$
\begin{aligned}
& \Delta_{a c}^{+}-\Delta_{a c}^{-}=-1,0 \leq \Delta_{a c}^{+}, \Delta_{a c}^{-} \leq 1 \Rightarrow \Delta_{a c}^{+}=0, \Delta_{a c}^{-}=1 \\
& \Delta_{b c}^{+}-\Delta_{b c}^{-}=-1,0 \leq \Delta_{b c}^{+}, \Delta_{b c}^{-} \leq 1 \Rightarrow \Delta_{b c}^{+}=0, \Delta_{b c}^{-}=1
\end{aligned}
$$

Take ac as an instance. Its change over $W_{p}$ and $W_{c}$ is $\Delta_{a c}^{+}-\Delta_{a c}^{-}=-1$, and both $\Delta_{a c}^{+}$and $\Delta_{a c}^{-}$are bounded by 0 and 1 ; therefore, the only possibility is that $\Delta_{a c}^{+}=$ 0 and $\Delta_{a c}^{-}=1$. Further, by applying $\mathrm{R}_{3}$ and $\mathrm{R}_{4}$, one has the following facts:

$$
\begin{array}{ll}
\Delta_{a b c}^{+} \leq \Delta_{a c}^{+}=0 & \Rightarrow \Delta_{a b c}^{+}=0 \\
\Delta_{a b c}^{-} \geq \Delta_{a c}^{-}+\Delta_{b c}^{-}-\Delta_{c}^{-}=1 & \Rightarrow \Delta_{a b c}^{-}=1
\end{array}
$$

Take abc as an instance. Following the inclusion-exclusion principle, one knows that $\Delta_{a b c}^{+}$should be no greater than $\Delta_{a c}^{+}=0$; hence, $\Delta_{a b c}^{+}=0$. Meanwhile, $\Delta_{a b c}^{-}$ has tight upper and lower bounds as 1 . The estimation of abc over $W_{c}$ is thus given by $T_{W_{c}}(a b c)=T_{W_{p}}(a b c)+\Delta_{a b c}^{+}-\Delta_{a b c}^{-}=3$, and the $\mathcal{P}_{h v} \overline{a b c}$ is uncovered.

The computation overhead of inter-window inference is dominated by the cost of solving the constrained integer optimization problems. The available fast off-theshelf tools make such attack feasible even with moderate computation power.

\section{OVERVIEW OF BUTTERFLY*}

Motivated by the inferencing attack model above, we outline ButTerflY*, our solution to protecting output privacy for (stream) mining applications.

\subsection{Design Objective}

Alternative to the reactive, detecting-then-removing scheme, we intend to use a proactive approach to tackle both intra-window and inter-window inference in a uniform manner. Our approach is motivated by two key observations. First, in many mining applications, the utility of mining output are measured by metrics other than the exact support of individual itemsets, but rather the semantic relationship of their support (e.g., the ordering or ratio of support values). It is thus acceptable to trade the precision of individual itemsets for boosting the outputprivacy guarantee, provided that the desired output utility is maintained. Second, both intra-window and inter-window inferencing attacks are based on the inclusionexclusion principle, which involves multiple frequent itemsets. Trivial randomness injected into each frequent itemset can accumulate into considerable uncertainty in inferred patterns. The more complicated the inference (i.e., harder to detect), the more considerable such uncertainty.

We therefore propose BUTTERFLY*, a light-weighted output-privacy preservation scheme based on pattern perturbation. By sacrificing certain trivial precision of individual frequent itemsets, it significantly amplifies the uncertainty of vulnerable patterns, thus blocking both intra-window and inter-window inference.

\subsection{Mining Output Perturbation}

Data perturbation refers to the process of modifying confidential data while preserving its utility for intended applications [Adam and Worthmann 1989]. This is arguably the most important technique used to date for protecting original input 
data. In our scheme, we employ perturbation to inject uncertainty into mining output. The perturbation over output pattern significantly differs from that over input data. In input perturbation, the data utility is defined by the overall statistical characteristics of dataset. The distorted data is fed as input into the following mining process. Typically no utility constraints are attached to individual data values. While in output perturbation, the perturbed results are directly presented to end-users, and the data utility is defined over each individual value.

There are typically two types of utility constraints for the perturbed results. First, each reported value should have enough accuracy, i.e., the perturbed value should not deviate widely from the actual value. Second, the semantic relationships among the results should be preserved to the maximum extent. There exist nontrivial trade-offs among these utility metrics. To our best knowledge, this work is the first to consider such multiple trade-offs in mining output perturbation.

Concretely, we consider the following two perturbation techniques, with their roots at statistics literature [Adam and Worthmann 1989; Chin and Ozsoyoglu 1981]: value distortion perturbs the support by adding a random value drawn from certain probabilistic distribution; value bucketization partitions the range of support into a set of disjoint, mutually exclusive intervals. Instead of reporting the exact support, one returns the interval which the support belongs to.

Both techniques can be applied to output perturbation. However, value bucketization leads to fairly poor utility compared with value distortion, since all support values with in an interval are modified to the same value, and any semantic constraints, e.g., order or ratio, can hardly be enforced in this model. We thus focus on value distortion in the following discussion. Moreover, in order to guarantee the precision of each individual frequent itemset, we are more interested in probabilistic distributions with bounded intervals. We thus exemplify with a discrete uniform distribution over integers, although our discussion is applicable for other distributions (details in Section 7).

\subsection{Operation of ButTERFLY*}

On releasing the mining output of a stream window, one perturbs the support of each frequent itemset $X, T(X)^{1}$ by adding a random variable $r_{X}$ drawn from a discrete uniform distribution over integers within an interval $\left[l_{X}, u_{X}\right]$. The sanitized support $T^{\prime}(X)=T(X)+r_{X}$ is hence a random variable, which can be specified by its bias $\beta(X)$ and variance $\sigma^{2}(X)$. Intuitively, the bias indicates the difference of the expected value $E\left[T^{\prime}(X)\right]$ and the actual value $T(X)$, while the variance represents the average deviation of $T^{\prime}(X)$ from $E\left[T^{\prime}(X)\right]$. Note that compared with $T(X)$, $r_{X}$ is non-significant, i.e., $\left|r_{X}\right| \ll T(X)$.

While this operation is simple, the setting of $\beta(X)$ and $\sigma^{2}(X)$ is non-trivial, in order to achieve sufficient privacy protection and utility guarantee simultaneously, which is the focus of our following discussion. Specifically, we will address the tradeoff between privacy guarantee and output utility in Section 5, and the trade-offs among multiple utility metrics in Section 6 .

\footnotetext{
${ }^{1}$ In what follows, without ambiguity, we omit the referred database $\mathcal{D}$ in the notations.
} 


\section{BASIC BUTTERFLY*}

We start with defining the metrics to quantitatively measure the precision of individual frequent itemsets, and the privacy protection for vulnerable patterns.

\subsection{Precision Measure}

The precision loss of a frequent itemset $X$ incurred by perturbation can be measured by the mean square error (mse) of the perturbed support $T^{\prime}(X)$ :

$$
\operatorname{mse}(X)=E\left[\left(T^{\prime}(X)-T(X)\right)^{2}\right]=\sigma^{2}(X)+\beta^{2}(X)
$$

Intuitively, mse $(X)$ measures the average deviation of perturbed support $T^{\prime}(X)$ with respect to actual value $T(X)$. A smaller mse implies higher accuracy of the output. Also, it is conceivable that the precision loss should take account of the actual support. The same mse may indicate sufficient accuracy for an itmeset with large support, but may render the output of little value for an itemset with small support. Therefore, we have the following precision metric:

Definition 5.1. (Precision Degradation) For each frequent itemset $X$, its precision degradation, denoted by $\operatorname{pred}(X)$, is defined as the relative mean squared error of $T^{\prime}(X)$ :

$$
\operatorname{pred}(X)=\frac{E\left[\left(T^{\prime}(X)-T(X)\right)^{2}\right]}{T^{2}(X)}=\frac{\sigma^{2}(X)+\beta^{2}(X)}{T^{2}(X)}
$$

\subsection{Privacy Measure}

Distorting the original support of frequent itemsets is only a part of the story, it is necessary to ensure that the distortion cannot be filtered out. Hence, one needs to consider the adversary's power in estimating the support of vulnerable patterns through the protection.

Without loss of generality, assume that the adversary desires to estimate the support of pattern $P$ of the form $I \oplus(\overline{J \ominus I})$, and has full access to the sanitized support $T^{\prime}(X)$ of all $X \in \mathcal{X}_{I}^{J}$. Let $T^{\prime \prime}(P)$ denote the adversary's estimation regarding $T(P)$. The privacy protection should be measured by the error of $T^{\prime \prime}(P)$. Following let us discuss such estimation from the adversary's perspective. Along the discussion, we will show how various prior knowledge possessed by the adversary may impact the estimation.

Recall that $T(p)$ is estimated following the inclusion-exclusion principle: $T(p)=$ $\sum_{X \in \mathcal{X}_{I}^{J}}(-1)^{|X \ominus I|} T(X)$. From the adversary's view, each support $T(X)\left(X \in \mathcal{X}_{I}^{J}\right)$ is now a random variable; $T(P)$ is thus also a random variable. The estimation accuracy of $T^{\prime \prime}(P)$ with respect to $T(P)$ (by the adversary) can be measured by the mean square error, defined as mse $(P)=E\left[\left(T(P)-T^{\prime \prime}(P)\right)^{2}\right]$. We consider the worst case (the best case for the adversary) wherein mse $(P)$ is minimized, and define the privacy guarantee based on this lower bound. Intuitively, a larger min mse $(P)$ indicates a more significant error in estimating $T(P)$ by the adversary, and thus better privacy protection. Also it is noted that the privacy guarantee should account for actual support $T(P)$ : if $T(P)$ is close to zero, trivial variance makes it hard for the adversary to infer if pattern $P$ exists. Such "zero-indistinguishability" decreases as $T(P)$ grows. Therefore, we introduce the following privacy metric for vulnerable pattern $P$. 
Definition 5.2. (PrivaCy Guarantee) For each vulnerable pattern $P$, its privacy guarantee, denoted by $\operatorname{prig}(P)$, is defined as its minimum relative estimation error (by the adversary):

$$
\operatorname{prig}(P)=\frac{\min \operatorname{mse}(P)}{T^{2}(P)}
$$

In the following, we show how various assumptions regarding the adversary's prior knowledge impact this privacy guarantee. We start the analysis by considering each itemset independently, then take account of the interrelations among them.

Prior Knowledge 5.3. The adversary may have full knowledge regarding the applied perturbation, including its distribution and parameters.

In our case, the parameter of $r_{X}$ specifies the interval $\left[l_{X}, r_{X}\right]$ from which the random variable $r_{X}$ is drawn; therefore, from the adversary's view, of each $X \in \mathcal{X}_{I}^{J}$, its actual support $T(X)=T^{\prime}(X)-r_{X}$, is a random variable following a discrete uniform distribution over interval $\left[l_{X}^{\prime}, u_{X}^{\prime}\right]$, where $l_{X}^{\prime}=T^{\prime}(X)-u_{X}, u_{X}^{\prime}=T^{\prime}(X)-l_{X}$ and has expectation $T^{\prime}(X)-\left(l_{X}+u_{X}\right) / 2$ and variance $\sigma^{2}(X)$. Recalling that $\left|r_{X}\right| \ll T(X)$, this is a bounded distribution over positive integers. Given the expectation of each $T(X)$, we have the following theorem that dictates the lower bound of $\operatorname{mse}(P)$.

Theorem 5.4. Given the distribution $f(x)$ of a random variable $x$, the mean square error of an estimate $e$ of $x, \operatorname{mse}(e)=\int_{-\infty}^{\infty}(x-e)^{2} f(x) d x$ reaches its minimum value $\operatorname{Var}[x]$, when $e=E[x]$.

Proof (TheOREM 5.4). We have the following derivation:

$$
\begin{aligned}
\operatorname{mse}(x) & =\int_{-\infty}^{\infty}(x-e)^{2} f(x) \mathrm{d} x \\
& =E\left[x^{2}\right]+e^{2}-2 e \cdot E[x] \\
& =(e-E[x])^{2}+\operatorname{Var}[x]
\end{aligned}
$$

Hence, mse $(e)$ is minimized when $e=E[x]$.

Therefore, mse $(P)$ is minimized when $T^{\prime \prime}(P)=E[T(P)]$, which is the best guess the adversary can achieve (note that the optimality is defined in terms of average estimation error, not the semantics, e.g., $E[T(P)]$ is possibly negative). In this best case for the adversary, the lowest estimation error is reached as $\operatorname{Var}[T(P)]$.

In the case that each itemset is considered independently, the fact that $T(p)$ is a linear combination of all involved $T(X)$ implies that $\operatorname{Var}[T(p)]$ can be approximated by the sum of the variance of all involved $T(X)$, i.e., min $\operatorname{mse}(p)=\sum_{X \in \mathcal{X}_{I}^{J}} \sigma^{2}(X)$.

Prior Knowledge 5.5. The support values of different frequent itemsets are interrelated by a set of inequalities, derived from the inclusion-exclusion principle.

Here, we take into consideration the dependency among the involved itemsets. As we have shown, each itemset $X$ is associated with an interval $\left[l_{X}^{\prime}, u_{X}^{\prime}\right]$ containing its possible support. Given such itemset-interval pairs, the adversary may attempt to apply these inequalities to tighten the intervals, and thus obtaining better estimation regarding the support. Concretely, this idea can be formalized in the entailment problem [Calders 2004]:

ACM Transactions on Database Systems, Vol. , No. , 20 
Definition 5.6 (Entailment). A set of itemset-interval pairs $\mathcal{C}$ entail a constraint $T(X) \in\left[l_{X}, u_{X}\right]$, denoted by $\mathcal{C}=T(X) \in\left[l_{X}, u_{X}\right]$ if every database $\mathcal{D}$ that satisfies $\mathcal{C}$, also satisfies $T(X) \in\left[l_{X}, u_{X}\right]$. The entailment is tight if for every smaller interval $\left[l_{X}^{\prime}, u_{X}^{\prime}\right] \subset\left[l_{X}, u_{X}\right], \mathcal{C} \not \models T(X) \in\left[l_{X}^{\prime}, u_{X}^{\prime}\right]$, i.e., $\left[l_{X}, u_{X}\right]$ is the best interval that can be derived for $T(X)$ based on $\mathcal{C}$.

Clearly, the goal of the adversary is to identify the tight entailment for each $T(X)$ based on the rest; however, we have the following complexity result.

Theorem 5.7. Deciding whether $T(X) \in\left[l_{X}, u_{X}\right]$ is entailed by a set of itemsetinterval pairs $\mathcal{C}$ is DP-Complete.

Proof (Theorem 5.7-Sketch). Deciding whether $\mathcal{C} \models T(X) \in\left[l_{X}, u_{X}\right]$ is equivalent to the entailment problem in the context of probabilistic logic programming with conditional constraints [Lukasiewicz 2001], which is proved to be DPComplete.

This theorem indicates that it is hard to leverage the dependency among the involved itemsets to improve the estimation of each individual itemset; therefore, one can approximately consider the support values of frequent itemsets as independent varaibles in measuring the adversary's power. The privacy guarantee $\operatorname{prig}(P)$ can thus be expressed as $\operatorname{prig}(P)=\sum_{X \in \mathcal{X}_{I}^{J}} \sigma^{2}(X) / T^{2}(P)$.

Prior Knowledge 5.8. The adversary may have access to other forms of prior knowledge, e.g., published statistics of the dataset, samples of a similar dataset, or support of the top-k frequent itemsets, etc.

All these forms of prior knowledge can be captured by the notion of knowledge point: a knowledge point is a specific frequent itemset $X$, for which the adversary has estimation error less than $\sigma^{2}(X)$. Note that following Theorem 5.7, the introduction of knowledge points in general does not influence the estimation of the other itemsets. Our definition of privacy guarantee can readily incorporate this notion. Concretely, let $\mathcal{K}_{I}^{J}$ denote the set of knowledge points in the set of $\mathcal{X}_{I}^{J}$, and $\kappa^{2}(X)$ be the average estimation error of $T(X)$ for $X \in \mathcal{K}_{I}^{J}$. We therefore have the refined definition of privacy guarantee.

$$
\operatorname{prig}(P)=\frac{\sum_{X \in \mathcal{K}_{I}^{J}} \kappa^{2}(X)+\sum_{X \in \mathcal{X}_{I}^{J} \backslash \mathcal{K}_{I}^{J}} \sigma^{2}(X)}{T^{2}(P)}
$$

Another well-known uncertainty metric is entropy. Both variance and entropy are important and independent measures of privacy protection. However, as pointed out in [Hore et al. 2004], variance is more appropriate in measuring individualcentric privacy wherein the adversary is interested in determining the precise value of a random variable. We therefore argue that variance is more suitable for our purpose, since we are aiming at protecting the exact support of vulnerable patterns.

Prior Knowledge 5.9. The sanitized support of the same frequent itemsets may be published in consecutive stream windows.

Since our protection is based on independent random perturbation, if the same support value is repeatedly perturbed and published in multiple windows, the adversary can potentially improve the estimation by averaging the observed output 
(the law of large numbers). To block this type of attack, once the perturbed support of a frequent itemset is released, we keep publishing this sanitized value if the actual support remains unchanged in consecutive windows.

Discussion. In summary, the effectiveness of ButTERFLY* is evaluated in terms of its resilience against both intra-window and inter-window inference over stream mining output. We note three key implications.

First, the uncertainty of involved frequent itemsets is accumulated in the inferred vulnerable patterns. Moreover, more complicated inferencing attacks (i.e., harder to be detected) face higher uncertainty.

Second, the actual support of a vulnerable pattern is typically small (only a unique or less than $K$ records match vulnerable patterns), and thus adding trivial uncertainty can make it hard to tell the existence of this pattern in the dataset.

Third, inter-window inference follows a two-stage strategy, i.e., first deducing the transition between contingent windows, then inferring the vulnerable patterns. The uncertainty associated with both stages provides even stronger protection.

\subsection{Trade-off between Precision and Privacy}

In our ButTERfl* framework, the tradeoff between privacy protection and output utility can be flexibly adjusted by the setting of variance and bias for each frequent itemset. Specifically, variance controls the overall balance between privacy and utility, while bias gives a finer control over the balance between precision and other utility metrics, as we will show later. Here, we focus on the setting of variance. Intuitively, smaller variance leads to higher output precision, however also decreases the uncertainty of inferred vulnerable patterns, thus lower privacy guarantee.

To ease the discussion, we assume that all the frequent itemsets are associated with the same variance $\sigma^{2}$ and bias $\beta$. In Section 6 when semantic constraints are taken into account, we will lift this simplification, and consider more sophisticated settings.

Let $C$ denote the minimum support for frequent itemsets. From the definition of precision metrics, it can be derived that for each frequent itemset $X$, its precision degradation $\operatorname{pred}(X) \leq\left(\sigma^{2}+\beta^{2}\right) / C^{2}$, because $T(X) \geq C$. Let $P_{1}(C)=\left(\sigma^{2}+\right.$ $\left.\beta^{2}\right) / C^{2}$, i.e., the upper bound of precision loss for frequent itemsets. Meanwhile, for a vulnerable pattern $P=I(\overline{J \backslash I})$, it can be proved that its privacy guarantee $\operatorname{prig}(P) \geq\left(\sum_{X \in \mathcal{X}_{I}^{J}} \sigma^{2}\right) / K^{2} \geq\left(2 \sigma^{2}\right) / K^{2}$, because $T(P) \leq K$ and the inference involves at least two frequent itemsets. Let $P_{2}(C, K)=\left(2 \sigma^{2}\right) / K^{2}$, i.e., the lower bound of privacy guarantee for inferred vulnerable patterns.

$P_{1}$ and $P_{2}$ provide convenient representation to control the trade-off. Specifically, setting an upper bound $\epsilon$ over $P_{1}$ guarantees sufficient accuracy of the reported frequent itemsets; while setting a lower bound $\delta$ over $P_{2}$ provides enough privacy protection for the vulnerable patterns. One can thus specify the precision-privacy requirement as a pair of parameters $(\epsilon, \delta)$, where $\epsilon, \delta>0$. That is, the setting of $\beta$ and $\sigma$ should satisfy $P_{1}(C) \leq \epsilon$ and $P_{2}(C, K) \geq \delta$, as

$$
\begin{gathered}
\sigma^{2}+\beta^{2} \leq \epsilon C^{2} \\
\sigma^{2} \geq \delta K^{2} / 2
\end{gathered}
$$

To make both inequalities hold, it should be satisfied that $\epsilon / \delta \geq K^{2} /\left(2 C^{2}\right)$. The 
term $\epsilon / \delta$ is called the precision-privacy ratio (PPR). When precision is a major concern, one can set PPR as its minimum value $K^{2} /\left(2 C^{2}\right)$ for given $K$ and $C$, resulting in the minimum precision loss for given privacy requirement. The minimum PPR also implies that $\beta=0$ and the two parameters $\epsilon$ and $\delta$ are coupled. We refer to the perturbation scheme with the minimum PPR as the basic ButTERFLY* scheme.

\section{OPTIMIZED BUTTERFLY*}

The basic ButTerfLY* scheme attempts to minimize the precision loss of individual frequent itemsets, without taking account of their semantic relationships. Although easy to implement and resilient against attacks, this simple scheme may easily violate these semantic constraints directly related to the specific applications of the mining output, and thus decreasing the overall utility of the results. In this section, we refine this basic scheme by taking semantic constraints into our map, and develop constraint-aware ButTeRFLY* schemes. For given precision and privacy requirement, the optimized scheme preserves the utility-relevant semantics to the maximum extent.

In this work, we specifically consider two types of constraints, absolute ranking and relative frequency. By absolute ranking, we refer to the order of frequent itemsets according to their support. In certain applications, users pay special attention to the ranking of patterns, rather than their actual support, e.g., querying the topten most popular purchase patterns. By relative frequency, we refer to the pair-wise ratio of the support of frequent itemsets. In certain applications, users care more about the ratio of two frequent patterns, instead of their absolute support, e.g., computing the confidence of association rules.

To facilitate the presentation, we first introduce the concept of frequency equivalent class (FEC).

Definition 6.1. (Frequent Equivalent Class). A frequent equivalent class (FEC) is a set of frequent itemsets that feature equivalent support. Two itemsets $I, J$ belong to the same FEC if and only if $T(I)=T(J)$. The support of a FEC $f e c, T(f e c)$, is defined as the support of any of its members.

A set of frequent itemsets can be partitioned into a set of disjoint FECs, according to their support. Also note that a set of FECs are a strictly ordered sequence: we define two FECs $f e c_{i}$ and $f e c_{j}$ as $f e c_{i}<f e c_{j}$ if $T\left(f e c_{i}\right)<T\left(f e c_{j}\right)$. Following we assume that the given set of FECs $\mathcal{F E C}$ are sorted according to their support, i.e., $T\left(f e c_{i}\right)<T\left(f e c_{j}\right)$ for $i<j$.

Example 6.2. In our running example as shown in Fig. 3, given $C=4$, there are three FECs, $\{c d\},\{a c, b c\},\{c\}$, with support 4,5 and 8, respectively.

Apparently, to comply with the constraints of absolute ranking or relative frequency, the equivalence of itemsets in a FEC should be preserved to the maximum extent in the perturbed output. Thus, in our constraint-aware schemes, the perturbation is performed at the level of FECs, instead of each specific itemset.

We argue that this change does not affect the privacy guarantee as advertised, provided the fact that the inference of a vulnerable pattern involves at least two 
frequent itemsets with different support, i.e., at least two FECs. Otherwise assuming that the involved frequent itemsets belong to the same FEC, the inferred vulnerable pattern would have support zero, which is a contradiction. Therefore, as long as each FEC is associated with uncertainty satisfying Eq.(2), the privacy preservation is guaranteed to be above the advertised threshold.

\subsection{Order Preservation}

When the order of itemset support is an important concern, the perturbation of each FEC cannot be uniform, since that would easily invert the order of two itemsets, especially when their support values are close. Instead, one needs to maximally separate the perturbed support of different FECs, under the given constraints of Eq.(1) and Eq.(2). To capture this intuition, we first introduce the concept of uncertainty region of FEC.

Definition 6.3. (UNCERTAINTY REGION) The uncertainty region of FEC $f e c$ is the set of possible values of its perturbed support: $\left\{x \mid \operatorname{Pr}\left(T^{\prime}(f e c)=x\right)>0\right\}$.

For instance, when adding to FEC fec a random variable drawn from a discrete uniform distribution over interval $[a, b]$, the uncertainty region is all the integers within interval $[a+T(f e c), b+T(f e c)]$. To preserve the order of FECs with overlapping uncertainty regions, we maximally reduce their intersection, by adjusting their bias setting.

Example 6.4. As shown in Fig. 4, three FECs have intersected uncertainty regions, and their initial biases are all zero. After adjusting the biases properly, they share no overlapping uncertainty region; thus, the order of their support is preserved in the perturbed output.

Note that the order is not guaranteed to be preserved if some FECs still have overlapping regions after adjustment, due to the constraints of given precision and privacy parameters $(\epsilon, \delta)$. We intend to achieve the maximum preservation under the given requirement.

Minimizing Overlapping Uncertainty Region. Below we formalize the problem of order preservation. Without loss of generality, consider two FECs $f e c_{i}, f e c_{j}$ with $T\left(f e c_{i}\right)<T\left(f e c_{j}\right)$. To simplify the notation, we use the following short version: let $t_{i}=T\left(f e c_{i}\right), t_{j}=T\left(f e c_{j}\right), t_{i}^{\prime}$ and $t_{j}^{\prime}$ be their perturbed support, and $\beta_{i}$ and $\beta_{j}$ the bias setting, respectively.

The order of $f e c_{i}$ and $f e c_{j}$ can be possibly inverted if their uncertainty regions intersect; that is, $\operatorname{Pr}\left[t_{i}^{\prime} \geq t_{j}^{\prime}\right]>0$. We attempt to minimize this inversion probability $\operatorname{Pr}\left[t_{i}^{\prime} \geq t_{j}^{\prime}\right]$ by adjusting $\beta_{i}$ and $\beta_{j}$. This adjustment is not arbitrary, constrained by the precision and privacy requirement. We thus introduce the concept of maximum adjustable bias:

Definition 6.5. (Maximum Adjustable Bias) For each FEC fec, its bias is allowed to be adjusted within the range of $\left[-\beta_{\max }(f e c), \beta_{\max }(f e c)\right], \beta_{\max }(f e c)$ is called the maximum adjustable bias. For given $\epsilon$ and $\delta$, it is defined as

$$
\beta_{\max }(f e c)=\left\lfloor\sqrt{\epsilon T^{2}(f e c)-\delta K^{2} / 2}\right\rfloor
$$

derived from Eq.(1) and Eq.(2).

ACM Transactions on Database Systems, Vol. , No. , 20 


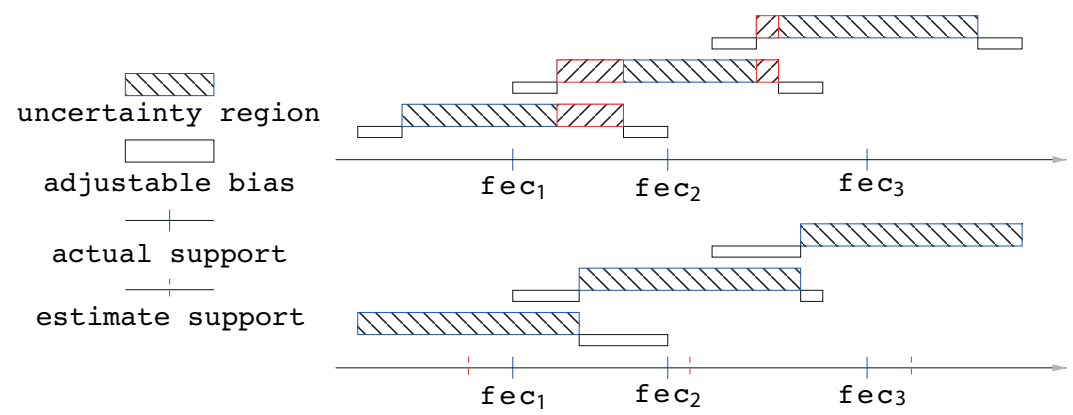

Fig. 4. Adjusting bias to minimize overlapping uncertainty regions.

Wrapping up the discussion above, the problem of preserving absolute ranking can be formalized as: Given a set of FECs $\left\{f e c_{1}, \ldots, f e c_{n}\right\}$, find the optimal bias setting for each FEC fec within its maximum adjustable bias $\left[-\beta_{\max }(f e c), \beta_{\max }(f e c)\right]$ to minimize the sum of pair-wise inversion probability: $\min \sum_{i<j} \operatorname{Pr}\left[t_{i}^{\prime} \geq t_{j}^{\prime}\right]$.

Exemplifying with a discrete uniform distribution, we now show how to compute $\operatorname{Pr}\left[t_{i}^{\prime} \geq t_{j}^{\prime}\right]$. Consider a discrete uniform distribution over interval $[a, b]$, with $\alpha$ $=b-a$ as the interval length. The variance of this distribution is given by $\sigma^{2}=$ $\left[(\alpha+1)^{2}-1\right] / 12$. According to Eq.(2) in Section 5, we have $\alpha=\left\lceil\sqrt{1+6 \delta K^{2}}\right\rceil-1$. Let $d_{i j}$ be the distance of their estimators $e_{i}=t_{i}+\beta_{i}$ and $e_{j}=t_{j}+\beta_{j}{ }^{2}$, i.e., $d_{i j}=e_{j}-e_{i}$.

The intersection of uncertainty regions of $f e c_{i}$ and $f e c_{j}$ is a piece-wise function, with four possible types of relationships: 1) $e_{i}<e_{j}, f e c_{i}$ and $f e c_{j}$ do not overlap; 2) $e_{i} \leq e_{j}, f e c_{i}$ and $f e c_{j}$ intersect; 3) $e_{i}>e_{j}, f e c_{i}$ and $f e c_{j}$ intersect; 4) $e_{i}>e_{j}$, $f e c_{i}$ and $f e c_{j}$ do not overlap. Correspondingly, the inversion probability $\operatorname{Pr}\left[t_{i}^{\prime} \geq t_{j}^{\prime}\right]$ is computed as follows:

$$
\operatorname{Pr}\left[t_{i}^{\prime} \geq t_{j}^{\prime}\right]= \begin{cases}0 & d_{i j} \geq \alpha+1 \\ \frac{\left(\alpha+1-d_{i j}\right)^{2}}{2(\alpha+1)^{2}} & 0<d_{i j}<\alpha+1 \\ 1-\frac{\left(\alpha+1+d_{i j}\right)^{2}}{2(\alpha+1)^{2}} & -\alpha-1<d_{i j} \leq 0 \\ 1 & d_{i j} \leq-\alpha-1\end{cases}
$$

Following we use $C_{i j}$ (or $C_{j i}$ ) to denote $\operatorname{Pr}\left[t_{i}^{\prime} \geq t_{j}^{\prime}\right]$, the cost function of the pair $f e c_{i}$ and $f e c_{j}$. The formulation of $C_{i j}$ can be considerably simplified based on the next key observation: for any pair $f e c_{i}$ and $f e c_{j}$ with $i<j$, the solution of the optimization problem contains no configuration of $d_{i j}<0$, as proved in the next lemma.

Lemma 6.6. In the optimization solution of min $\sum_{i<j} C_{i j}$, any pair of FECs fec $c_{i}$ and $f_{e c}$ with $i<j$ must have $e_{i} \leq e_{j}$, i.e., $d_{i j} \geq 0$.

Proof (Lemma 6.6). Assume that the estimators $\left\{e_{1}, \ldots, e_{n}\right\}$ corresponding to the optimal setting, and there exists a pair of FECs, $f e c_{i}$ and $f e c_{j}$ with $i<j$ and $e_{i}>e_{j}$. By switching their setting, i.e., let $e_{i}^{\prime}\left(\beta_{i}^{\prime}\right)$, and $e_{j}^{\prime}\left(\beta_{j}^{\prime}\right)$ be their new

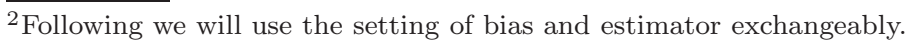


setting, and $e_{i}^{\prime}=e_{j}$, and $e_{j}^{\prime}=e_{i}$, the overall cost is reduced, because $\sum_{k \neq i, j} C_{k i}+$ $C_{k j}$ remains the same, and $C_{i j}$ is reduced, thus contradictory to the optimality assumption.

We need to prove that the new setting is feasible, that is $\left|\beta_{i}^{\prime}\right| \leq \beta_{\max }\left(\right.$ fec $\left._{i}\right)$ and $\left|\beta_{j}^{\prime}\right| \leq \beta_{\max }\left(f e c_{j}\right)$. Here, we prove the feasibility of $\beta_{i}^{\prime}$, and a similar proof applies to $\beta_{j}^{\prime}$. First, according to the assumption, we know that

$$
e_{j}=t_{j}+\beta_{j}<t_{i}+\beta_{i}=e_{i} \quad \text { and } \quad t_{i}<t_{j}
$$

therefore, we have the next fact:

$$
\beta_{i}^{\prime}=\beta_{j}+t_{j}-t_{i}<\beta_{i} \leq \beta_{\max }\left(f e c_{i}\right)
$$

We now just need to prove that $\beta_{i}^{\prime} \geq-\beta_{\max }\left(f e c_{i}\right)$, equivalent to $\beta_{j}+t_{j}-t_{i} \geq$ $-\beta_{\max }\left(f e c_{i}\right)$, which is satisfied if

$$
t_{j}-t_{i} \geq \beta_{\max }\left(f e c_{j}\right)-\beta_{\max }\left(f e c_{i}\right)
$$

By substituting the maximum adjustable bias with its definition, and considering the fact $\epsilon \leq 1$, this inequality can be derived.

Therefore, it is sufficient to consider the case $d_{i j} \geq 0$ for every pair of $f e c_{i}$ and $f e c_{j}$ when computing the inversion probability $\operatorname{Pr}\left[t_{i}^{\prime} \geq t_{j}^{\prime}\right]$. The optimization problem is thus simplified as: $\sum_{i<j}\left(\alpha+1-d_{i j}\right)^{2}$.

One flaw of the discussion so far is that we treat all FECs uniformly without considering their characteristics, i.e., the number of frequent itemsets within each FEC. The inversion of FECs containing more frequent itemsets is more serious than that of FECs with less members. Quantitatively, let $s_{i}$ be the number of frequent itemsets in the FEC $f e c_{i}$, the inversion of two FECs $f e c_{i}$ and $f e c_{j}$ means the ordering of $s_{i}+s_{j}$ itemsets are disturbed.

Therefore, our aim now is to solve the weighted optimization problem:

$$
\begin{array}{ll}
\min & \sum_{i<j}\left(s_{i}+s_{j}\right)\left(\alpha+1-d_{i j}\right)^{2} \\
\text { s.t. } & d_{i j}= \begin{cases}\alpha+1 & e_{j}-e_{i} \geq \alpha+1 \\
e_{j}-e_{i} & e_{j}-e_{i}<\alpha+1\end{cases} \\
& \forall i<j, e_{i} \leq e_{j} \\
& \forall i, e_{i} \in \mathbb{Z}^{+},\left|e_{i}-t_{i}\right| \leq \beta_{\max }\left(f e c_{i}\right)
\end{array}
$$

This is a quadratic integer programming (QIP) problem, with piece-wise cost function. In general, QIP is NP-Hard, even without integer constraints [Vavasis 1990]. This problem can be solved by first applying quadratic optimization techniques, such like simulated annealing, and then using random rounding techniques to impose the integer constraints. However, we are more interested in online algorithms that can flexibly trade between efficiency and accuracy. Following we present such a solution based on dynamic programming.

A Near Optimal Solution. By relaxing the constraint that $\forall i<j, e_{i} \leq e_{j}$ to $e_{i}<e_{j}$, we obtain the following key properties: (i) the estimators of all the FECs are in strict ascending order, i.e., $\forall i<j, e_{i}<e_{j}$; (ii) the uncertainty regions of all 
the FECs have the same length $\alpha$. Each FEC can thus intersect with at most $\alpha$ of its previous ones. These properties lead to an optimal substructure, crucial for our solution.

Lemma 6.7. Given that the biases of the last $\alpha$ FECs $\left\{f e c_{n-\alpha+1}: f e c_{n}\right\}^{3}$ are fixed as $\left\{\beta_{n-\alpha+1}: \beta_{n}\right\}$, and $\left\{\beta_{1}: \beta_{n-\alpha}\right\}$ are optimal w.r.t. $\left\{f e c_{1}: f e c_{n}\right\}$, then for given $\left\{\beta_{n-\alpha}: \beta_{n-1}\right\},\left\{\beta_{1}: \beta_{n-\alpha-1}\right\}$ must be optimal w.r.t. $\left\{\right.$ fec $_{1}:$ fec $\left._{n-1}\right\}$.

Proof (Lemma 6.7). Suppose that there exists a better setting $\left\{\beta_{1}^{\prime}: \beta_{n-\alpha-1}^{\prime}\right\}$ leading to lower cost w.r.t. $\left\{f e c_{1}: f e c_{n-1}\right\}$. Since $f e c_{n}$ does not intersect with any $\left\{f e c_{1}: f e c_{n-\alpha-1}\right\}$, the setting $\left\{\beta_{1}^{\prime}: \beta_{n-\alpha-1}^{\prime}, \beta_{n-\alpha}: \beta_{n}\right\}$ leads to lower cost w.r.t. $\left\{f e c_{1}: f e c_{n}\right\}$, contradictory to our optimality assumption.

Based on this optimal substructure, we propose a dynamic programming solution, which adds FECs sequentially according to their order. Let $C_{n-1}\left(\beta_{n-\alpha}: \beta_{n-1}\right)$ represent the minimum cost that can be achieved by adjusting FECs $\left\{f e c_{1}: f e c_{n-\alpha-1}\right\}$ with the setting of the last $\alpha$ FECs fixed as $\left\{\beta_{n-\alpha}: \beta_{n}\right\}$. When adding $f e c_{n}$, the minimum cost $C_{n}\left(\beta_{n-\alpha+1}: \beta_{n}\right)$ is computed using the rule:

$$
C_{n}\left(\beta_{n-\alpha+1}: \beta_{n}\right)=\min _{\beta_{n-\alpha}} C_{n-1}\left(\beta_{n-\alpha}: \beta_{n-1}\right)+\sum_{i=n-\alpha}^{n-1}\left(s_{i}+s_{n}\right)\left(\alpha+1-d_{i n}\right)^{2}
$$

The optimal setting is the one with the minimum cost among all the combination of $\left\{\beta_{n-\alpha+1}: \beta_{n}\right\}$.

Now, let us analyze the complexity of this scheme. Let $\beta_{\max }^{*}$ denote the maximum value of maximum adjustable biases of all FECs: $\beta_{\max }^{*}=\max _{i} \beta_{\max }\left(f e c_{i}\right)$. For each $f e c$, its bias can be chosen from at most $2 \beta_{\text {max }}^{*}+1$ integers. Computing $C_{n}\left(\beta_{n-\alpha+1}\right.$ : $\left.\beta_{n}\right)$ for each combination of $\left\{\beta_{n-\alpha+1}: \beta_{n}\right\}$ from $C_{n-1}\left(\beta_{n-\alpha}: \beta_{n-1}\right)$ takes at most $2 \beta_{\text {max }}^{*}+1$ steps, and the number of combinations is at most $\left(2 \beta_{\max }^{*}+1\right)^{\alpha}$. The time complexity of this scheme is thus bounded by $\left(2 \beta_{\max }^{*}+1\right)^{\alpha+1} n$, i.e., $O(n)$ where $n$ is the total number of FECs. Meanwhile, the space complexity is also bounded by the number of cost function values needed to be recorded for each FEC, i.e., $\left(2 \beta_{\max }^{*}+1\right)^{\alpha}$. In addition, at each step, we need to keep track of the bias setting for the added FECs so far for each combination, thus $\left(2 \beta_{\max }^{*}+1\right)^{\alpha}(n-\alpha)$ in total.

In practice, the complexity is typically much lower than this bound, given that (i) under the constraint $\forall i<j, e_{i}<e_{j}$, a number of combinations are invalid, and (ii) $\beta_{\max }^{*}$ is an over-estimation of the average maximum adjustable bias.

It is noted that as $\alpha$ or $\beta_{\max }^{*}$ grows, the complexity increases sharply, even though it is linear in terms of the total number of FECs. In view of this, we develop an approximate version of this schem that allows trading between efficiency and accuracy. The basic idea is that on adding each FEC, we only consider its intersection with its previous $\gamma$ FECs, instead of $\alpha$ ones $(\gamma<\alpha)$. This approximation is tight when the distribution of FECs is not extremely dense, which is usually the case, as verified by our experiments. Formally, a $(\gamma / \alpha)$-approximate solution is defined as:

$$
C_{n}\left(\beta_{n-\gamma+1}: \beta_{n}\right)=\min _{\beta_{n-\gamma}} C_{n-1}\left(\beta_{n-\gamma}: \beta_{n-1}\right)+\sum_{i=n-\gamma}^{n-1}\left(s_{i}+s_{n}\right)\left(\alpha+1-d_{i n}\right)^{2}
$$

${ }^{3}$ In the following we use $\left\{x_{i}: x_{j}\right\}$ as a short version of $\left\{x_{i}, x_{i+1}, \ldots, x_{j}\right\}$. 
Input: $\left\{t_{i}, \beta_{\max }\left(f e c_{i}\right)\right\}$ for each $f e c_{i} \in \mathcal{F E C}, \alpha, \gamma$.

Output: $\beta_{i}$ for each $f e c_{i} \in \mathcal{F E C}$.

begin

/*initialization*/;

for $\beta_{1}=-\beta_{\max }\left(f e c_{1}\right): \beta_{\max }\left(f e c_{1}\right)$ do

$\mathrm{L} C_{1}\left(\beta_{1}\right)=0$;

for $i=2: \gamma$ do

for $\beta_{i}=-\beta_{\max }\left(f e c_{i}\right): \beta_{\max }\left(f e c_{i}\right)$ do

$/ * e_{i}<e_{j} * /$

if $\beta_{i}+t_{i}>\beta_{i-1}+t_{i-1}$ then

$\left\lfloor C_{i}\left(\beta_{1}: \beta_{i}\right)=C_{i-1}\left(\beta_{1}: \beta_{i-1}\right)+\sum_{j=1}^{i-1}\left(s_{j}+s_{i}\right)\left(\alpha+1-d_{j i}\right)^{2} ;\right.$

/* dynamic programming $* /$;

for $i=\gamma+1: n$ do

for $\beta_{i}=-\beta_{\max }\left(f e c_{i}\right): \beta_{\max }\left(f e c_{i}\right)$ do

if $\beta_{i}+t_{i}>\beta_{i-1}+t_{i-1}$ then

$C_{i}\left(\beta_{i-\gamma+1}: \beta_{i}\right)=\min _{\beta_{i-\gamma}} C_{i-1}\left(\beta_{i-\gamma}: \beta_{i-1}\right)+$

$\sum_{j=i-\gamma}^{i-1}\left(s_{j}+s_{i}\right)\left(\alpha+1-d_{j i}\right)^{2}$

/*find the optimal setting*/;

find the minimum $C_{n}\left(\beta_{n-\gamma+1}: \beta_{n}\right)$;

backtrack and output $\beta_{i}$ for each $f e c_{i} \in \mathcal{F E C}$;

end

Algorithm 1: Order-preserving bias setting

Now the complexity is bounded by $\left(2 \beta_{\max }^{*}+1\right)^{\gamma+1} n$. By properly adjusting $\gamma$, one can control the balance between accuracy and efficiency.

The complete algorithm is sketched in Algorithm 1: one first initializes the cost function for the first $\gamma$ FECs; then by running the dynamic programming procedure, one computes the cost function for each newly added FEC. The optimal configuration is the one with the global minimum value $C_{n}\left(\beta_{n-\gamma+1}: \beta_{n}\right)$.

\subsection{Ratio Preservation}

In certain applications, the relative frequency of the support of two frequent itemsets carries important semantics, e.g., the confidence of association rules. However, the random perturbation may easily render the ratio of the perturbed support considerably deviate from the original value. Again, we achieve the maximum ratio preservation by intelligently adjust the bias setting of FECs. First, we formalize the problem of ratio preservation.

Maximizing $(k, 1 / k)$ Probability of Ratio. Consider two FECs $f e c_{i}$ and $f e c_{j}$ with $t_{i}<t_{j}$. To preserve the ratio of $f e c_{i}$ and $f e c_{j}$, one is interested in making the ratio of perturbed support $t_{i}^{\prime} / t_{j}^{\prime}$ appear in the proximate area of original value $t_{i} / t_{j}$ with high probability, e.g., interval $\left[k \frac{t_{i}}{t_{j}}, \frac{1}{k} \frac{t_{i}}{t_{j}}\right]$, where $k \in(0,1)$, indicating the tightness of this interval. We therefore introduce the concept of $(k, 1 / k)$ probability.

ACM Transactions on Database Systems, Vol. , No. , 20. 
Definition 6.8. $((k, 1 / k)$ Probability $)$ The $(k, 1 / k)$ probability of the ratio of two random variables $t_{i}^{\prime}$ and $t_{j}^{\prime}, \operatorname{Pr}_{(k, 1 / k)}\left[\frac{t_{i}^{\prime}}{t_{j}^{\prime}}\right]$ is defined as

$$
\operatorname{Pr}_{(k, 1 / k)}\left[\frac{t_{i}^{\prime}}{t_{j}^{\prime}}\right]=\operatorname{Pr}\left[k \frac{t_{i}}{t_{j}} \leq \frac{t_{i}^{\prime}}{t_{j}^{\prime}} \leq \frac{1}{k} \frac{t_{i}}{t_{j}}\right]
$$

This $(k, 1 / k)$ probability quantitatively describes the proximate region of original ratio $t_{i} / t_{j}$. A higher probability that $t_{i}^{\prime} / t_{j}^{\prime}$ appears in this region indicates better preservation of the ratio. The problem of ratio preservation is therefore formalized as the following optimization problem:

$$
\begin{array}{ll}
\max & \sum_{i<j} \operatorname{Pr}_{(k, 1 / k)}\left[\frac{t_{i}^{\prime}}{t_{j}^{\prime}}\right] \\
\text { s.t } & \forall i, e_{i} \in \mathbb{Z}^{+},\left|e_{i}-t_{i}\right| \leq \beta_{\max }\left(f e c_{i}\right)
\end{array}
$$

It is not hard to see that in the case of discrete uniform distribution, the $(k, 1 / k)$ probability of the ratio of two random variables is a non-linear piece-wise function, i.e., a non-linear integer optimization problem. In general, non-linear optimization problem is NP-Hard, even without integer constraints. Instead of applying off-theshelf non-linear optimization tools, we are more interested in efficient heuristics that can find near-optimal configurations with linear complexity in terms of the number of FECs. Following, we present one such scheme that performs well in practice.

A Near Optimal Solution. We construct our bias setting scheme based on Markov's Inequality. To maximize the $(k, 1 / k)$ probability $\operatorname{Pr}\left[k \frac{t_{i}}{t_{j}} \leq \frac{t_{i}^{\prime}}{t_{j}^{\prime}} \leq \frac{1}{k} \frac{t_{i}}{t_{j}}\right]$, we can alternatively minimize the probability $\operatorname{Pr}\left[\frac{t_{i}^{\prime}}{t_{j}^{\prime}} \geq \frac{1}{k} \frac{t_{i}}{t_{j}}\right]+\operatorname{Pr}\left[\frac{t_{j}^{\prime}}{t_{i}^{\prime}} \geq \frac{1}{k} \frac{t_{j}}{t_{i}}\right]$. From Markov's Inequality, we knows that the probability $\operatorname{Pr}\left[\frac{t_{i}^{\prime}}{t_{j}^{\prime}} \geq \frac{1}{k} \frac{t_{i}}{t_{j}}\right]$ is bounded by

$$
\operatorname{Pr}\left[\frac{t_{i}^{\prime}}{t_{j}^{\prime}} \geq \frac{1}{k} \frac{t_{i}}{t_{j}}\right] \leq \frac{E\left[\frac{t_{i}^{\prime}}{t_{j}^{\prime}}\right]}{\frac{1}{k} \frac{t_{i}}{t_{j}}}=k \frac{t_{j}}{t_{i}} E\left[\frac{t_{i}^{\prime}}{t_{j}^{\prime}}\right]
$$

The maximization of $(k, 1 / k)$ probability of $t_{i}^{\prime} / t_{j}^{\prime}$ is therefore simplified as the following expression ( $k$ is omitted since it does not affect the optimization result):

$$
\min \frac{t_{j}}{t_{i}} E\left[\frac{t_{i}^{\prime}}{t_{j}^{\prime}}\right]+\frac{t_{i}}{t_{j}} E\left[\frac{t_{j}^{\prime}}{t_{i}^{\prime}}\right]
$$

The intuition here is that neither expectation $\frac{t_{j}}{t_{i}} E\left[\frac{t_{i}^{\prime}}{t_{j}^{\prime}}\right]$ nor $\frac{t_{i}}{t_{j}} E\left[\frac{t_{j}^{\prime}}{t_{i}^{\prime}}\right]$ should deviate far from one.

According to its definition, the expectation of $\frac{t_{i}^{\prime}}{t_{j}^{\prime}}, E\left[\frac{t_{i}^{\prime}}{t_{j}^{\prime}}\right]$, is computed as

$$
E\left[\frac{t_{i}^{\prime}}{t_{j}^{\prime}}\right]=\frac{1}{(\alpha+1)^{2}} \sum_{e_{j}-\alpha / 2}^{e_{j}+\alpha / 2} \frac{1}{t_{j}^{\prime}} \sum_{e_{i}-\alpha / 2}^{e_{i}+\alpha / 2} t_{i}^{\prime}=\frac{e_{i}}{\alpha+1}\left(H_{e_{j}+\frac{\alpha}{2}}-H_{e_{j}-\frac{\alpha}{2}}\right)
$$


where $H_{n}$ is the $n$th harmonic number. It is known that $H_{n}=\ln n+\Theta(1)$, thus

$$
E\left[\frac{t_{i}^{\prime}}{t_{j}^{\prime}}\right] \approx \frac{e_{i}}{\alpha+1} \ln \frac{e_{j}+\alpha / 2}{e_{j}-\alpha / 2}=\frac{e_{i}}{\alpha+1} \ln \left(1+\frac{\alpha}{e_{j}-\alpha / 2}\right)
$$

This form is still not convenient for computation. We are therefore looking for a tight approximation for the logarithm part of the expression. It is known that $\forall x, y \in \mathbb{R}^{+},(1+x / y)^{y+x / 2}$ is a tight upper bound for $e^{x}$. We have the following approximation by applying this bound: $1+\alpha /\left(e_{j}-\alpha / 2\right)=e^{\frac{\alpha}{e_{j}-\alpha / 2+\alpha / 2}}=e^{\frac{\alpha}{e_{j}}}$.

Applying the approximation to computing $E\left[\frac{t_{i}^{\prime}}{t_{j}^{\prime}}\right]$ in Eq.(4), it is derived that

$$
E\left[\frac{t_{i}^{\prime}}{t_{j}^{\prime}}\right]=\frac{e_{i}}{\alpha+1} \ln e^{\frac{\alpha}{e_{j}}}=\frac{\alpha}{\alpha+1} \frac{e_{i}}{e_{j}}
$$

The optimization of Eq.(3) is thus simplified as

$$
\min \frac{t_{j}}{t_{i}} \frac{e_{i}}{e_{j}}+\frac{t_{i}}{t_{j}} \frac{e_{j}}{e_{i}}
$$

Assuming that $e_{i}$ is fixed, by differentiating Eq.(5) w.r.t. $e_{j}$, and setting the derivative as 0 , we get the solution of $e_{j}$ as $e_{j} / e_{i}=t_{j} / t_{i}$, i.e., $\beta_{j} / \beta_{i}=t_{j} / t_{i}$.

Following this solution is our bottom-up bias setting scheme: for each FEC fec $c_{i}$, its bias $\beta_{i}$ should be set in proportion to its support $t_{i}$. Note that the larger $t_{i}+\beta_{i}$ compared with $\alpha$, the more accurate the applied approximation applied; hence, $\beta_{i}$ should be set as its maximum possible value.

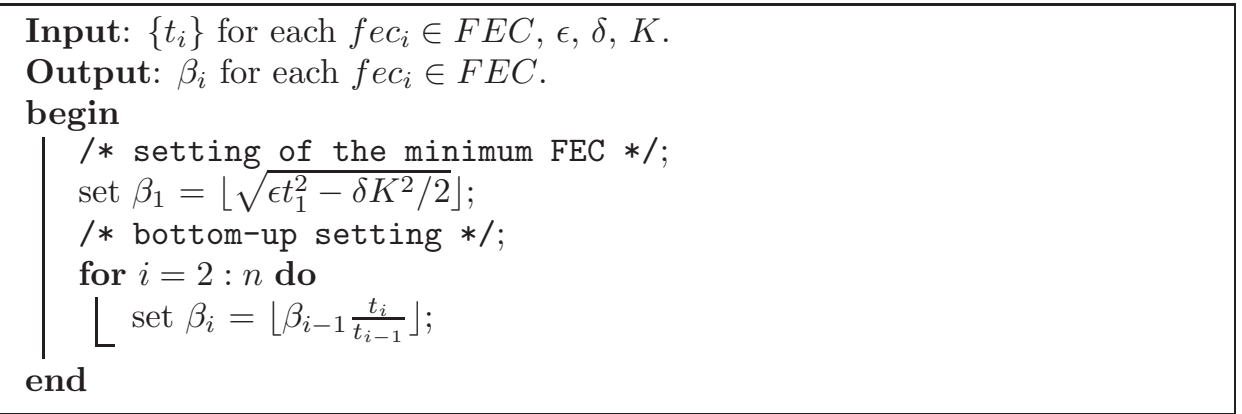

Algorithm 2: Ratio-preserving bias setting

Algorithm 2 sketches the bias setting scheme: one first sets the bias of the minimum FEC $f e c_{1}$ as its maximum $\beta_{\max }\left(f e c_{1}\right)$, and for each rest $\mathrm{FEC} f e c_{i}$, its bias $\beta_{i}$ is set in proportion to $t_{i} / t_{i-1}$. In this scheme, for any pair of $f e c_{i}$ and $f e c_{j}$, their biases satisfy $\beta_{i} / \beta_{j}=t_{i} / t_{j}$. Further, we have the following lemma to prove the feasibility of this scheme. By feasibility, we mean that for each FEC $f e c_{i}, \beta_{i}$ falls within the allowed interval $\left[-\beta_{\max }\left(f e c_{i}\right), \beta_{\max }\left(f e c_{i}\right)\right]$.

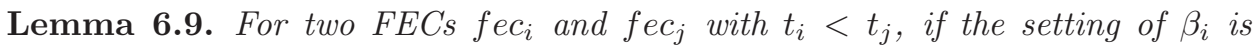
feasible for $f e c_{i}$, then the setting $\beta_{j}=\beta_{i} \frac{t_{j}}{t_{i}}$ is feasible for $\mathrm{fec}_{j}$.

ACM Transactions on Database Systems, Vol. , No. , 20. 
Proof (Lemma 6.9). Given that $0<\beta_{i} \leq \beta_{\max }\left(f e c_{i}\right)$, then according to the definition of maximum adjustable bias, $\beta_{j}$ has the following property

$$
\begin{aligned}
& \beta_{j}=\beta_{i} \frac{t_{j}}{t_{i}} \leq \beta_{\max }\left(f e c_{i}\right) \frac{t_{j}}{t_{i}}=\left\lfloor\sqrt{\epsilon t_{i}^{2}-\frac{\delta K^{2}}{2}}\right\rfloor \frac{t_{j}}{t_{i}} \\
& =\left\lfloor\sqrt{\epsilon t_{j}^{2}-\frac{\delta K^{2}}{2} \frac{t_{j}^{2}}{t_{i}^{2}}}\right\rfloor \leq\left\lfloor\sqrt{\epsilon t_{j}^{2}-\frac{\delta K^{2}}{2}}\right\rfloor=\beta_{\max }\left(f e c_{j}\right)
\end{aligned}
$$

Thus if $\beta_{1}$ is feasible for $f e c_{1}, \beta_{i}$ is feasible for any $f e c_{i}$ with $i>1$, since $t_{i}>t_{1}$.

\subsection{A Hybrid Scheme}

While order-preserving and ratio-preserving bias settings achieve the maximum utility at their ends, in certain applications wherein both semantic relationships are important, it is desired to balance the two factors in order to achieve the overall optimal quality.

We thus develop a hybrid bias setting scheme that takes advantage of the two schemes, and allows to flexibly adjust the trade-off between the two quality metrics. Specifically, for each FEC $f e c$, let $\beta_{o p}(f e c)$ and $\beta_{r p}(f e c)$ denote its bias setting obtained by the order-preserving and frequency-preserving schemes, respectively. We have the following setting based on a linear combination:

$$
\forall f e c \in \mathcal{F E C} \quad \beta(f e c)=\lambda \beta_{o p}(f e c)+(1-\lambda) \beta_{r p}(f e c)
$$

The parameter $\lambda$ is a real number within the interval of $[0,1]$, which controls the trade-off between the two quality metrics. Intuitively, a larger $\lambda$ tends to indicate more importance over order information, but less over ratio information, and vise versa. Particularly, the order-preserving and ratio-preserving schemes are the special cases when $\lambda=1$ and 0 , respectively.

\section{EXTENSION TO OTHER DISTRIBUTION}

In the section, we intend to study the impact of the perturbation distribution over the quality of privacy protection and (multi-)utility preservation. It will be revealed shortly that while uniform distributions lead to the best privacy protection, it may not be optimal in terms of other utility metrics.

\subsection{Privacy and Precision}

Recall that the precision degradation of frequent itemset $X$ is given by $\operatorname{pred}(X)=$ $\left[\sigma^{2}(X)+\beta^{2}(X)\right] / T^{2}(X)$, while the privacy guarantee of vulnerable pattern $P$ of the form $I \oplus(\overline{J \ominus I})$ is given by $\operatorname{prig}(P)=\sum_{X \in \mathcal{X}_{I}^{J}} \sigma^{2}(X) / T^{2}(P)$. Clearly, if two perturbation distributions share the same bias and variance, they offer the same amount of precision preservation for $X$ and privacy guarantee for $P$. Next we focus our discussion on order and ratio preservation.

\subsection{Order Preservation}

For ease of presentation, we assume that the perturbation added to the support of each FEC is drawn from a homogeneous distribution with probability density function $(\mathrm{PDF}) f(\cdot)$, plus a bias specific to this FEC. Following the development in Section 6, we attempt to minimize the sum of pair-wise inversion probability: 


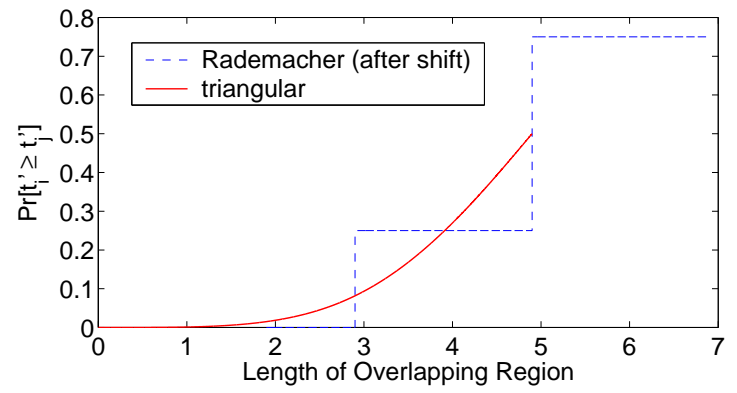

Fig. 5. Trade-off between uncertainty region length (intersection possibility) and probability mass density $(\sigma=1)$.

$\min \sum_{i<j} \operatorname{Pr}\left[t_{i}^{\prime} \geq t_{j}^{\prime}\right]$, by finding the optimal bias setting for each FEC $f e c_{i}$ within its maximum adjustable bias $\beta_{i}^{\max }$. Note that $\beta_{i}^{\max }=\left\lfloor\sqrt{\epsilon t_{i}^{2}-\delta K^{2} / 2}\right\rfloor$ is solely determined by the precision and privacy requirement $(\epsilon, \delta)$, irrespective of the underlying distribution $f(\cdot)$.

For general distribution $f(\cdot)$, the inversion probability $\operatorname{Pr}\left[t_{i}^{\prime} \geq t_{j}^{\prime}\right]$ is defined as:

$$
\begin{aligned}
\operatorname{Pr}\left[t_{i}^{\prime} \geq t_{j}^{\prime}\right] & =\int_{-\infty}^{+\infty} f\left(x_{j}-\beta_{j}\right) \int_{x_{j}+t_{j}-t_{i}}^{+\infty} f\left(x_{i}-\beta_{i}\right) \mathrm{d} x_{i} \mathrm{~d} x_{j} \\
& =\int_{-\infty}^{+\infty} f\left(x_{j}-\beta_{j}\right)\left(1-F\left(x_{j}+t_{j}-t_{i}-\beta_{i}\right)\right) \mathrm{d} x_{j} \\
& =\int_{-\infty}^{+\infty} F\left(x_{j}-\beta_{j}\right) f(x) \mathrm{d} x \\
& =\mathbb{E}\left[F\left(x-\left(t_{j}-t_{i}+\beta_{j}-\beta_{i}\right)\right)\right] \triangleq \mathbb{E}\left[F\left(x-d_{i j}\right)\right]
\end{aligned}
$$

where $F(\cdot)$ is the cumulative distribution function $(\mathrm{CDF})$ of $f(\cdot)$, and $d_{i j}$ denotes the distance of the estimators of $t_{i}$ and $t_{j}$. Clearly, $\operatorname{Pr}\left[t_{i}^{\prime} \geq t_{j}^{\prime}\right]$ is the expectation of the CDF after shifting transformation, which is a continuous function of $d_{i j}$ for unbounded distribution, e.g., normal distribution, and possibly piece-wise function for discrete distribution, e.g., uniform distribution; thus, no closed form of $\operatorname{Pr}\left[t_{i}^{\prime} \geq\right.$ $\left.t_{j}^{\prime}\right]$ is available for general $f(\cdot)$.

It is noted that Lemma 6.6 makes no specific assumption regarding the underlying distribution, and thus holding for any distribution $f(\cdot)$; therefore, under the optimal bias setting, for any $i<j$, it must hold that $d_{i j} \geq 0$. Furthermore, let $s_{i}$ be the number of frequent itemsets within the FEC $f e c_{i}$. Taking into consideration the weight of each FEC, the optimization problem is formulated as:

$$
\begin{array}{ll}
\min & \sum_{i<j}\left(s_{i}+s_{j}\right) \mathbb{E}\left[F\left(x-e_{j}+e_{i}\right)\right] \\
\text { s.t. } & \forall i,\left|e_{i}-t_{i}\right| \leq \beta_{i}^{\max } \\
& \forall i<j, e_{i} \leq e_{j}
\end{array}
$$

This is in general a non-linear programming problem, with the difficulty of optimization mainly depending on the concrete form of the underlying distribution. For 
example, in the case of uniform distribution, it becomes a quadratic programming problem (QIP); while in the case of Rademacher distribution, it becomes a piecewise minimization problem. Tailored optimization tools are therefore necessary for different distributions, which is beyond the scope of this work. Here, instead, we attempt to explore the interplay between the distribution of perturbation noise and that of itemset support.

From Fig. 4, it is noticed that two contributing factors affect the inversion probability $\operatorname{Pr}\left[t_{i}^{\prime} \geq t_{j}^{\prime}\right]$, namely, the length of the uncertainty regions of $f e c_{i}$ and $f e c_{j}$, and the average probability mass (per unit length) of $f e c_{i}$ and $f e c_{j}$ in the intersected region. Intuitively, if the uncertainty region length is large, the average probability mass distributed over the region tends to be small, but the possibility that two uncertainty regions intersect is high; meanwhile, if the uncertainty region length is small, they will have less chance to intersect, but the probability mass density in the intersected region will be large if they overlap. Here, we consider two representative distributions featuring small and large uncertainty regions for fixed variance $\sigma^{2}$.

- Rademacher distribution. Its probability mass function $f(\cdot)$ is defined as:

$$
f(x)=\left\{\begin{array}{cl}
1 / 2 & x=-\sigma \text { or } \sigma \\
0 & \text { otherwise }
\end{array}\right.
$$

The uncertainty region length is $2 \sigma$.

- triangular distribution. Its probability density function $f(\cdot)$ is given by:

$$
f(x)=\left\{\begin{array}{cl}
(\sqrt{6} \sigma+x) /\left(6 \sigma^{2}\right) & x \in[-\sqrt{6} \sigma, 0] \\
(\sqrt{6} \sigma-x) /\left(6 \sigma^{2}\right) & x \in[0, \sqrt{6} \sigma] \\
0 & \text { otherwise }
\end{array}\right.
$$

The uncertainty region length is $2 \sqrt{6} \sigma$.

Now, exemplifying with these two distributions, we attempt to explore the tradeoff of uncertainty region length and probability mass density that contribute to the inversion probability. Fig. 5 illustrates the inversion probability $\operatorname{Pr}\left[t_{i}^{\prime} \geq t_{j}^{\prime}\right]$ as a function of the intersection length of two uncertainty regions. To reflect the difference of uncertainty region length of the two distributions, we horizontally shift the inversion probability of Rademacher distribution $2(\sqrt{6}-1) \sigma$ units. It is noted that there is no clear winner over the entire interval; rather, each distribution demonstrates superiority over the other in certain regions. For example, when the intersection length is small, Rachemacher is better than triangular since its inversion probability is close to zero; when the intersection length reaches 3 , there is a sharp increase in the inversion probability of Rachemacher, which makes triangular a better choice; after the intersection length exceeds 4, triangular dominates Rachemacher in terms of the inversion probability again.

From the analysis above, we can conclude: 1) No single distribution is optimal for all possible distributions of support in terms of order preservation; rather, the perturbation distribution needs to be carefully selected, adapted to the underlying support distribution. 2) Intuitively, when the underlying support distribution is relative sparse, i.e., the gap between two consecutive support values is large, 


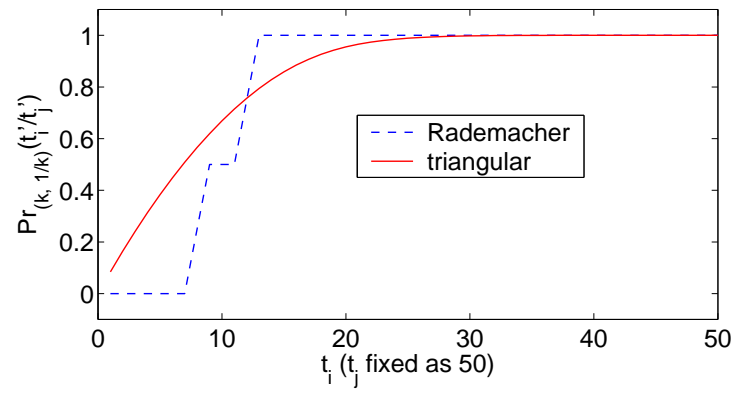

Fig. 6. Trade-off between uncertainty region length and probability mass density in ratio preservation, the parameter setting is: $\sigma=1, \beta_{i}=0, \beta_{j}=0$, and $t_{j}=50$.

distributions with small uncertainty regions, e.g., Rachmacher, are more preferable, which lead to less intersection possibility; when the support distribution is dense, distributions with less probability mass density, e.g., triangular, are more preferable. 3) The impact of the perturbation distribution over the quality of order preservation needs to empirically evaluated.

7.2.1 Ratio Preservation. Next we study the impact of perturbation distribution over the quality of ratio preservation. We first re-formulate the $(k, 1 / k)$ probability under general distribution $f(\cdot)$. For ease of presentation, we assume that the perturbation distributions for all FECs are homogeneous, plus a FEC-specific bias.

Under general distribution $f(\cdot)$, the $(k, 1 / k)$ probability is calculated as follows:

$$
\begin{aligned}
\operatorname{Pr}_{(k, 1 / k)}\left[t_{i}^{\prime} / t_{j}^{\prime}\right]= & \int_{-\infty}^{\infty} f\left(x_{j}-\beta_{j}\right) \int_{k \frac{t_{i}}{t_{j}}\left(t_{j}+x_{j}\right)-t_{i}}^{\frac{1}{t_{i}}\left(t_{j}+x_{j}\right)-t_{i}} f\left(x_{i}-\beta_{i}\right) \mathrm{d} x_{i} \mathrm{~d} x_{j} \\
= & \mathbb{E}\left[F\left(\frac{t_{i}}{t_{j}} \frac{x}{k}+\frac{t_{i}}{k}-t_{i}+\frac{t_{i}}{t_{j}} \frac{\beta_{j}}{k}-\beta_{i}\right)\right. \\
& \left.-F\left(k \frac{t_{i}}{t_{j}} x+k t_{i}-t_{i}+k \frac{t_{i}}{t_{j}} \beta_{j}-\beta_{i}\right)\right]
\end{aligned}
$$

Clearly, this quantity is the expectation difference of two CDFs after scaling and shifting transformation. Similar to the problem of order optimization, the difficulty of optimizing $\min \sum_{i<j} \operatorname{Pr}_{(k, 1 / k)}\left[t_{i}^{\prime} / t_{j}^{\prime}\right]$ depends on the concrete form of the underlying perturbation distribution $f(\cdot)$, which needs to be investigated on a case-by-case basis, and is beyond the scope of this work. Here, we are interested in investigating the impact of uncertainty region length and probability mass density over the $(k, 1 / k)$ probability.

Fig. 6 illustrates the trade-off between uncertainty region length and probability mass density, with respect to varying ratio of $t_{i} / t_{j}$. For presentation purpose, we filter out the effect of bias setting ( $\beta_{i}$ and $\beta_{j}$ are fixed as zero). We then fix $t_{j}$, and measure the $(k, 1 / k)$ probability $\operatorname{Pr}_{(k, 1 / k)}\left[t_{i}^{\prime} / t_{j}^{\prime}\right]$ of the two distributions, Rademacher and triangular, under varying $t_{i}$. Again, neither distribution demonstrates consistent superiority over the entire interval: for small ratio $t_{i} / t_{j}$, triangular is better than Rademacher given its larger $(k, 1 / k)$ probability; as the ratio increases, Rademacher offers better quality of ratio preservation; while for large 
ratio (close to 1), the influence of both distributions is non-significant.

We can thus draw conclusions similar to the case of order preservation: no single distribution is optimal for all possible support distributions in terms of ratio preservation; rather, the perturbation distribution needs to be selected, based on the underlying support distribution. A rule of thumb is: when the underlying support distribution is sparse, i.e., a large number of small ratios, distributions with small probability mass density, e.g., triangular, are more preferable; when the support distribution is relative dense, distributions with smaller uncertainty regions, e.g., Rademacher, are more preferable.

\section{EXPERIMENTAL ANALYSIS}

In this section, we investigate the efficacy of the proposed ButterflY* approaches. Specifically, the experiments are designed to measure the following three properties: 1) privacy guarantee: the effectiveness against both intra-window and inter-window inference; 2) result utility: the degradation of the output precision, the order and ratio preservation, and the trade-off among these utility metrics; 3) execution efficiency: the time taken to perform our approaches. We start with describing the datasets and the setup of the experiments.

\subsection{Experimental Setting}

We tested our solutions over both synthetic and real datasets. The synthetic dataset T20I4D50K is obtained by using the data generator as described in [Agrawal and Srikant 1994], which mimics transactions from retail stores. The real datasets used include: 1) BMS-WebView-1, which contains a few months of clickstream data from an e-commerce web site; 2) BMS-POS, which contains several years of point-of-sale from a large number of electronic retailers; 3) Mushroom in UCI KDD archive ${ }^{4}$, which is used widely in machine learning research. All these datasets have been used in frequent pattern mining over streams [Chi et al. 2006].

We built our ButterflY* prototype on top of Moment [Chi et al. 2006], a streaming frequent pattern mining framework, which finds closed frequent itemsets over a sliding window model. By default, the minimum support $C$ and vulnerable support $K$ are set as 25 and 5 , respectively, and the window size is set as $2 K$. Note that the setting here is designed to test the effectiveness of our approach with high ratio of vulnerable/minimum threshold $(K / C)$. All the experiments were performed over a workstation with Intel Xeon $3.20 \mathrm{GHz}$ and $4 \mathrm{~GB}$ main memory, running Red Hat Linux 9.0 operating system. The algorithm is implemented in $\mathrm{C}++$ and compiled using $\mathrm{g}++3.4$.

\subsection{Experimental Results}

To provide an in-depth understanding of our output-privacy preservation schemes, we evaluated four different versions of ButterfLY*: the basic version, the optimized version with $\lambda=0,0.4$, and 1 , respectively, over both synthetic and real datasets. Note that $\lambda=0$ corresponds to the ratio-preserving scheme, while $\lambda=1$ corresponds to the order-preserving one.

\footnotetext{
${ }^{4}$ http://kdd.ics.uci.edu/
} 


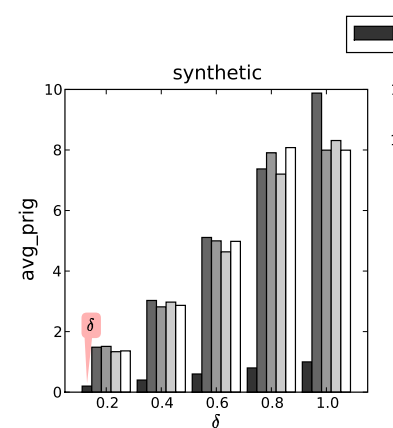

$\delta$ or $\epsilon \square$ basic $\square$ opt $(\lambda=1) \square$ opt $(\lambda=0) \square$ opt $(\lambda=0.4)$
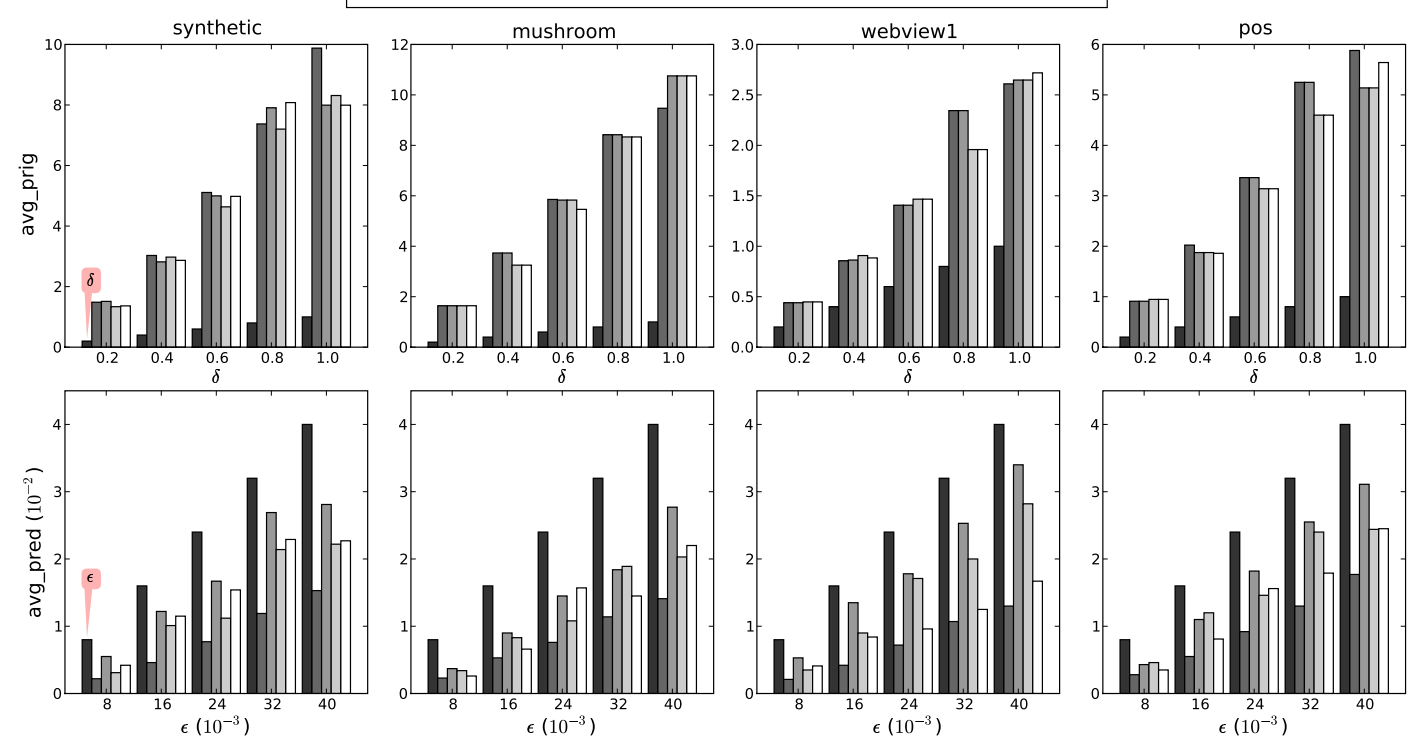

Fig. 7. Average privacy guarantee (avg_prig) and precision degradation (avg_pred).

Privacy and Precision. To evaluate the effectiveness of our approach in terms of output-privacy protection, we need to find all potential privacy breaches in the mining output. This is done by running an analysis program over the results returned by the mining algorithm, and finding all possible vulnerable patterns that can be inferred through either intra-window or inter-window inference.

Concretely, given a stream window, let $\mathcal{P}_{h v}$ denote all the hard vulnerable patterns that are inferable from the mining output. After the perturbation, we evaluate the relative deviation of the inferred value and the estimator for each pattern $P \in \mathcal{P}_{h v}$ for 100 continuous windows. we use the following average privacy (avg_prig) metric to measure the effectiveness of privacy preservation:

$$
\text { avg_prig }=\sum_{P \in \mathcal{P}_{h v}} \frac{\left(T^{\prime}(P)-E\left[T^{\prime}(P)\right]\right)^{2}}{T^{2}(P)\left|\mathcal{P}_{h v}\right|}
$$

The decrease of output precision is measured by the average precision degradation (avg_pred) of all frequent itemsets $\mathcal{I}$ :

$$
\text { avg_pred }=\sum_{I \in \mathcal{I}} \frac{\left(T^{\prime}(I)-T(I)\right)^{2}}{T^{2}(I)|\mathcal{I}|}
$$

In this set of experiments, we fix the precision-privacy ratio $\epsilon / \delta=0.04$, and measure avg_prig and avg_pred for different settings of $\epsilon(\delta)$.

Specifically, the four plots in the top tier of Fig. 7 show that as the value of $\delta$ increases, all four versions of ButTERFLF* provide similar amount of average privacy protection for all the datasets, far above the minimum privacy guarantee $\delta$. The four plots in the lower tier show that as $\sigma$ increases from 0 to 0.04 , the 
output precision decreases; however, all four versions of BuTTERFLY* have average precision degradation below the system-supplied maximum threshold $\epsilon$. Also note that among all the schemes, basic ButTerfLY* achieves the minimum precision loss, for given privacy requirement. This can be explained by the fact that the basic approach considers no semantic relationships, and sets all the bias as zero, while optimized Butterfly* trades precision for other utility-related metrics. Although the basic scheme maximally preserves the precision, it may not be optimal in the sense of other utility metrics, as shown next.

Order and Ratio. For given privacy and precision requirement $(\epsilon, \delta)$, we measure the effectiveness of ButTERFLY* in preserving order and ratio of frequent itemsets.

The quality of order preservation is evaluated by the proportion of order-preserved pairs over all possible pairs, referred to as the rate of order preserved pairs (ropp):

$$
\text { ropp }=\frac{\sum_{I, J \in \mathcal{I} \text { and } T(I) \leq T(J)} \mathbf{1}_{T^{\prime}(I) \leq T^{\prime}(J)}}{C_{|\mathcal{I}|}^{2}}
$$

where $\mathbf{1}_{x}$ is the indicator function, returning 1 if condition $x$ holds, and 0 otherwise.

Analogously, the quality of ratio preservation is evaluated by the fraction of the number of $(k, 1 / k)$ probability-preserved pairs over the number of possible pairs, referred to as the rate of ratio preserved pairs (rrpp) ( $k$ is set 0.95 in all the experiments):

$$
\operatorname{rrpp}=\frac{\sum_{I, J \in \mathcal{I} \text { and } T(I) \leq T(J)} \mathbf{1}_{k \frac{T(I)}{T(J)} \leq \frac{T^{\prime}(I)}{T^{\prime}(J)} \leq \frac{1}{k} \frac{T(I)}{T(J)}}}{C_{|\mathcal{I}|}^{2}}
$$

In this set of experiments, we vary the precision-privacy ratio $\epsilon / \delta$ for fixed $\delta=$ 0.4 , and measure the ropp and rrpp for four versions of BUTTERFLF* (the parameter $\gamma=2$ in all the experiments), as shown in Fig. 8.

As predicted by our theoretical analysis, the order-preserving $(\lambda=1)$ and ratiopreserving $(\lambda=0)$ bias settings are fairly effective, both outperform all other schemes at their ends. The ropp and rrpp increase as the ratio of $\epsilon / \delta$ grows, due to the fact that larger $\epsilon / \delta$ offers more adjustable bias therefore leading to better quality of order or ratio preservation.

It is also noticed that order-preserving scheme has the worst performance in the terms of avg_rrpp, even worse than the basic approach. This is explained by that in order to distinguish overlapping FECs, the order-preserving scheme may significantly distort the ratio of pairs of FECs. In all these cases, the hybrid scheme $\lambda=0.4$ achieves the second best in terms of avg_rrpp and avg_ropp, and an overall best quality when order and ratio preservation are equally important.

Tuning of Parameters $\gamma$ and $\lambda$. Next we give a detailed discussion on the setting of the parameters $\gamma$ and $\lambda$.

Specifically, $\gamma$ controls the depth of dynamic programming in the order-preserving bias setting. Intuitively, a larger $\gamma$ leads to better quality of order preservation, but also higher time and space complexity. We desire to characterize the gain of the quality of order preservation with respect to $\gamma$, and find the setting that balances the quality and efficiency.

For all four datasets, we measured the ropp with respect to the setting of $\gamma$, with 


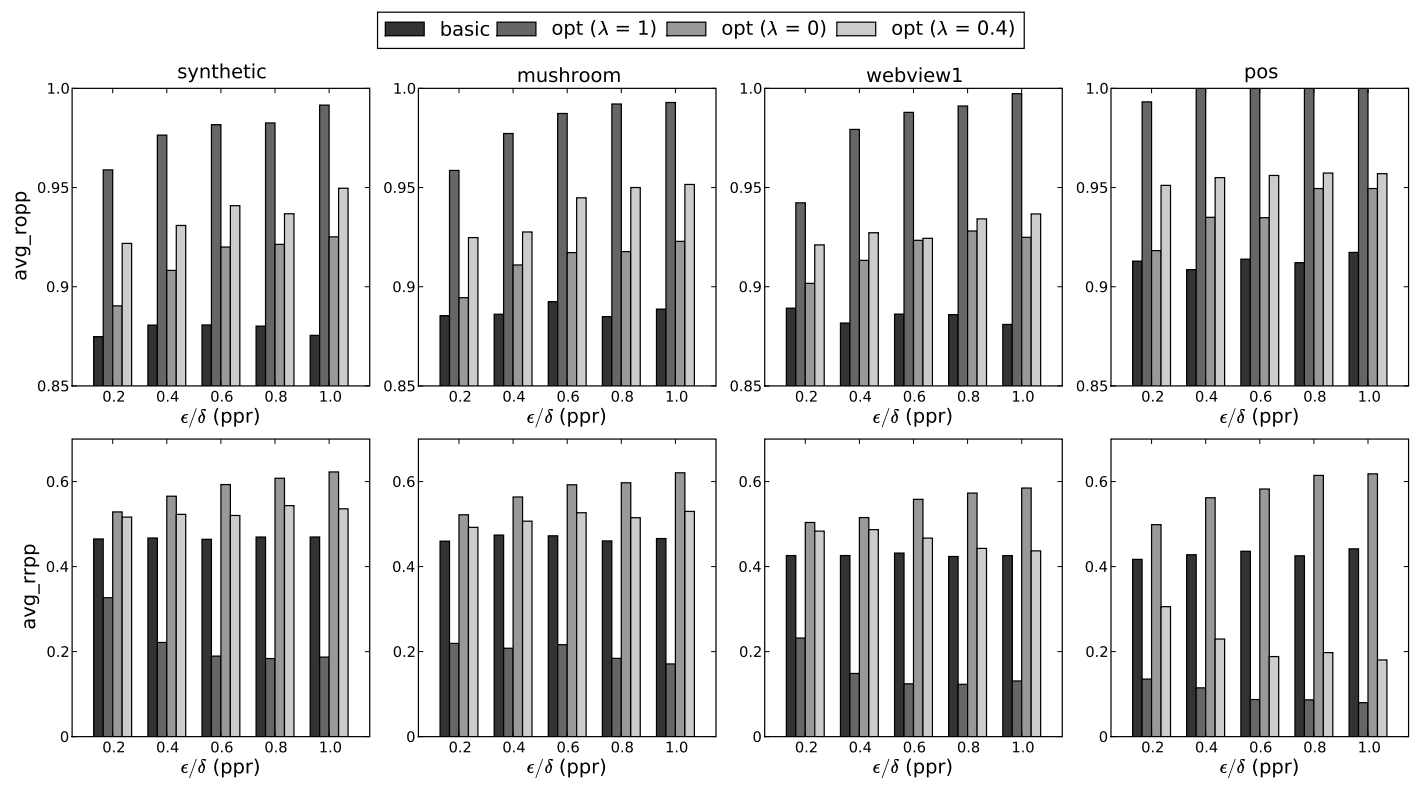

Fig. 8. Average order preservation (avg_ropp) and ratio preservation (avg_rrpp).
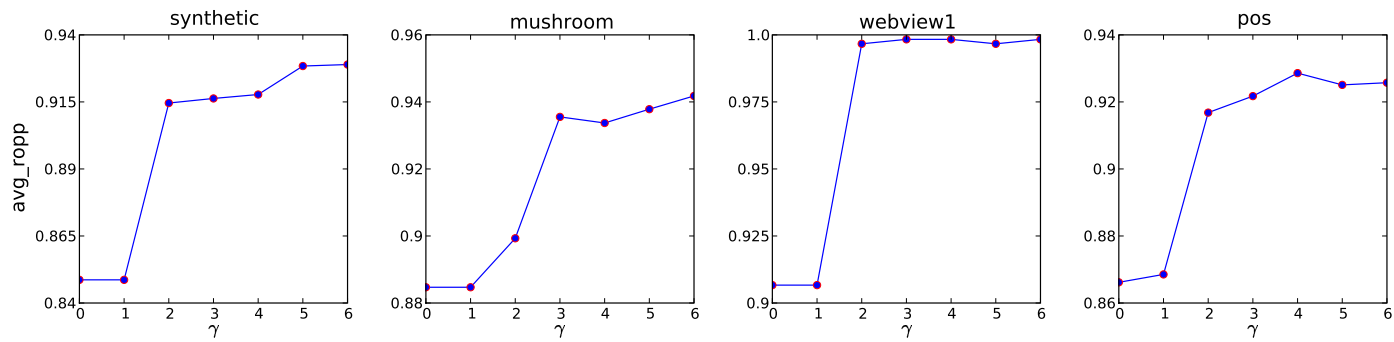

Fig. 9. Average rate of order-preserved pairs with respect to setting of $\gamma$.

result shown in Fig. 9. It is noted that the quality of order-preservation increases sharply at certain points $\gamma=2$ or 3 , and the trend becomes much flatter after that. This is explained by that in most real datasets, the distribution of FECs is not extremely dense; under proper setting of $(\epsilon, \delta)$, a FEC can intersect with only 2 or 3 neighboring FECs on average. Therefore, the setting of small $\gamma$ is usually sufficient for most datasets.

The setting of $\lambda$ balances the quality of order and ratio preservation. For each dataset, we evaluate ropp and rrpp with different settings of $\lambda(0.2,0.4,0.6,0.8$ and $1)$ and precision-privacy ratio $\epsilon / \delta(0.3,0.6$ and 0.9$)$, as shown in Fig. 10.

These plots give good estimation of the gain of order preservation, for given cost of ratio preservation that one is willing to sacrifice. A larger $\epsilon / \delta$ gives more room for this adjustment. In most cases, the setting of $\lambda=0.4$ offers a good balance between the two metrics. The trade-off plots could be made more accurate by ACM Transactions on Database Systems, Vol. , No. , 20. 

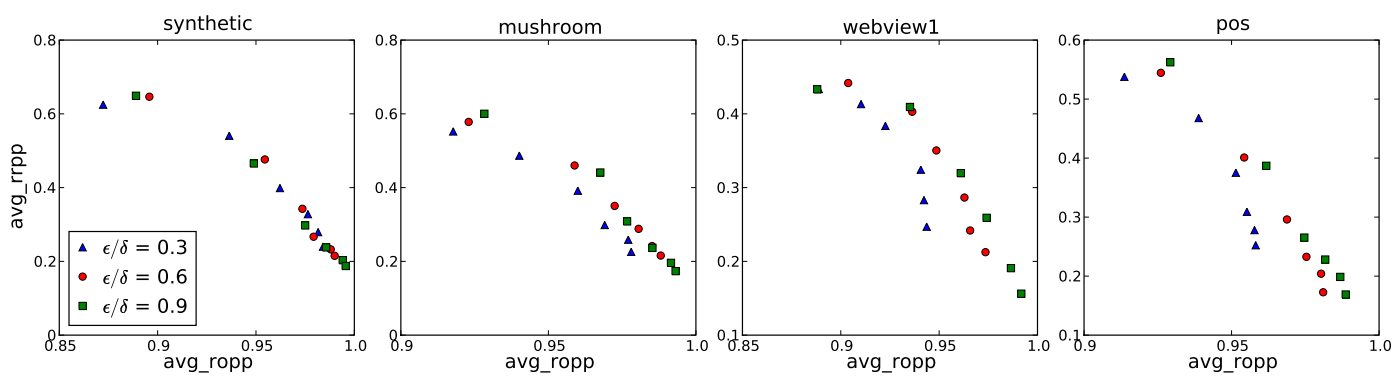

Fig. 10. Trade-off between order preservation and ratio preservation.

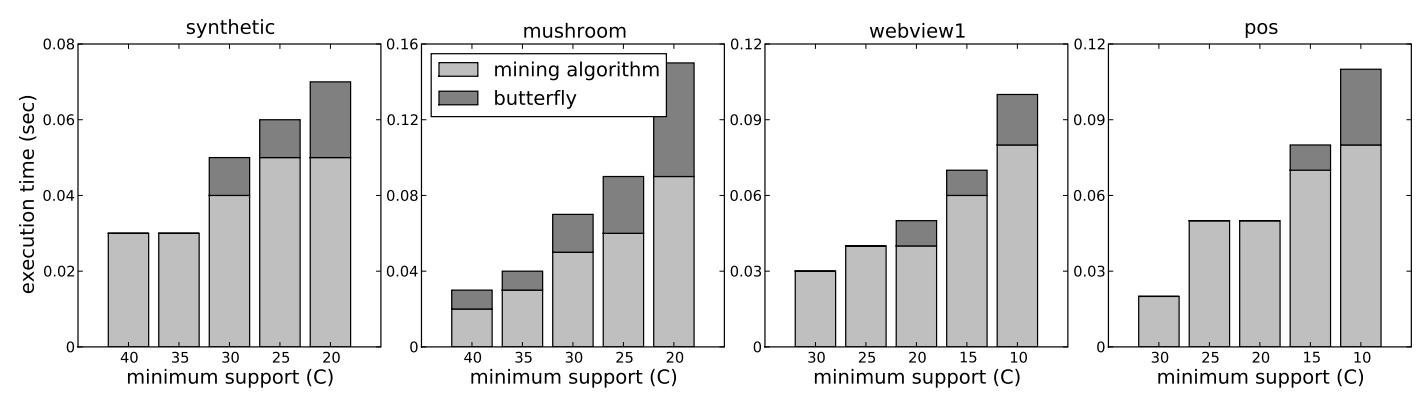

Fig. 11. Overhead of BUTTERFLY* algorithms in stream mining systems.

choosing more settings of $\lambda$ and $\epsilon / \delta$ to explore more points in the space.

8.2.1 Execution Efficiency. In the last set of experiments, we measured the computation overhead of BUTTERFLY* over the original mining algorithm for different settings of minimum support $C$. We divide the execution time into two parts contributed by the mining algorithm (mining algorithm) and BUTTERFLY algorithm (butterfly), respectively. Note that we do not distinguish basic and optimized Butterfly*, since basic Butterfly* involves simple perturbation operations, with unnoticeable cost. The window size is set $5 \mathrm{~K}$ for all four datasets.

The result plotted in Fig. 11 shows clearly that the overhead of Butterfly* is much less significant than the mining algorithm; therefore, it can be readily implemented in existing stream mining systems. Further, while the current versions of Butterfly* are window-based, it is expected that the incremental versions of ButTerfly* can achieve even lower overhead.

It is noted that in most cases, the running time of both mining algorithm and BUtTERFLY* algorithm grow significantly as $C$ decreases; however, the growth of the overhead of BUtTERFLY* is much less evident compared with the mining algorithm itself. This is expected since as the minimum support decreases, the number of frequent itemsets increases super-linearly, but the number of FECs has much lower growth rate, which is the most influential factor for the performance of BUTTERFLY*. 


\section{RELATED WORK}

\subsection{Disclosure Control in Statistical Database}

The most straightforward solution to preserving output privacy is to detect and eliminate all potential privacy breaches, i.e., the detecting-then-removing strategy, which stemmed from the inference control in statistical and census databases from 1970's. Motivated by the need of publishing census data, the statistics literature focuses mainly on identifying and protecting the privacy of sensitive data entries in contingency tables, or tables of counts corresponding to cross-classification of the microdata.

Extensive research has been done in statistical databases to provide statistical information without compromising sensitive information regarding individuals [Chin and Ozsoyoglu 1981; Shoshani 1982; Adam and Worthmann 1989]. The techniques, according to their application scenarios, can be broadly classified into query restriction and data perturbation. The query restriction family includes controlling the size of query results [Fellegi 1972], restricting the overlap between successive queries [Dobkin et al. 1979], suppressing the cells of small size [Cox 1980], and auditing queries to check privacy compromises [Chin and Ozsoyoglu 1981]; the data perturbation family includes sampling microdata [Denning 1980], swapping data entries between different cells [Dalenius and Reiss 1980], and adding noises to the microdata [Traub et al. 1984] or the query results [Denning 1980]. Data perturbation by adding statistical noise is an important method of enhancing privacy. The idea is to perturb the true value by a small amount $\epsilon$ where $\epsilon$ is a random variable with mean $=0$ and a small variance $=\sigma^{2}$. While we adopt the method of perturbation from statistical literature, one of our key technical contributions is the generalization of the basic scheme by adjusting the mean to accommodate various semantic constraints in the applications of mining output.

\subsection{Input Privacy Preservation}

Intensive research efforts have been directed to addressing the input-privacy issues. The work of [Agrawal and Srikant 2000; Agrawal and Aggarwal 2001] paved the way for the rapidly expanding field of privacy-preserving data mining; they established the main theme of privacy-preserving data mining as to provide sufficient privacy guarantee while minimizing the information loss in the mining output. Under this framework, a variety of techniques have been developed.

The work of [Agrawal and Srikant 2000; Agrawal and Aggarwal 2001; Evfimievski et al. 2002; Chen and Liu 2005] applied data perturbation, specifically random noise addition, to association rule mining, with the objective of maintaining sufficiently accurate estimation of frequent patterns while preventing disclosure of specific transactions (records). In the context of data dissemination and publication, group-based anonymization approaches have been considered. The existing work can be roughly classified as two categories: the first one aims at devising anonymization models and principles, as the criteria to measure the quality of privacy protection, e.g., $k$-anonymity [Sweeney 2002], $l$-diversity [Machanavajjhala et al. 2006], $(\epsilon, \delta)^{k}$-dissimilarity [Wang et al. 2009], etc.; the second category of work explores the possibility of fulfilling the proposed anonymization principles, meanwhile preserving the data utility to the maximum extent, e.g., [LeFevre et al. 2006;

ACM Transactions on Database Systems, Vol. , No. , 20 
Park and Shim 2007]. Cryptographic tools have also been used to construct privacypreserving data mining protocols, e.g., secure multi-party computation [Lindell and Pinkas 2000; Vaidya and Clifton 2002]. Nevertheless, all these techniques focus on protecting input privacy for static datasets, Quite recently, the work [Li et al. 2007] addresses the problem of preserving input privacy for streaming data, by online analysis of correlation structure of multivariate streams. The work [Bu et al. 2007] distinguishes the scenario of data custodian, where the data collector is entrusted, and proposes a perturbation scheme that guarantees no change in the mining output. In [Kargupta et al. 2003; Huang et al. 2005], it is shown that a hacker can potentially employ spectral analysis to separate the random noise from the real values for multi-attribute data.

\subsection{Output Privacy Preservation}

Compared with the wealth of techniques developed for preserving input privacy, the attention given to protecting mining output is fairly limited. The existing literature can be broadly classified as two categories. The first category attempts to propose general frameworks for detecting potential privacy breaches. For example, the work [Kantarcioğlu et al. 2004] proposes an empirical testing scheme for evaluating if the constructed classifier violates the privacy constraint. The second category focuses on proposing algorithms to address the detected breaches for specific mining tasks. For instance, it is shown in [Atzori et al. 2008] that the association rules can be exploited to infer information about individual transactions; while the work [Wang et al. 2007] proposes a scheme to block the inference of sensitive patterns satisfying user-specified templates by suppressing certain raw transactions. This paper is developed based on our previous work [Wang and Liu 2008].

\section{CONCLUSIONS}

In this work, we highlighted the importance of imposing privacy protection over (stream) mining output, a problem complimentary to conventional input privacy protection. We articulated a general framework of sanitizing sensitive patterns (models) to achieve output-privacy protection. We presented the inferencing and disclosure scenarios wherein the adversary performs attacks over the mining output. Motivated by the basis of the attack model, we proposed a lighted-weighted countermeasure, Butterfly*. It counters the malicious inference by amplifying the uncertainty of vulnerable patterns, at the cost of trivial decrease of output precision; meanwhile, for given privacy and precision requirement, it maximally preserves the utility-relevant semantics in mining output, thus achieving the optimal balance between privacy guarantee and output quality. The efficacy of ButTerfLF* is validated by extensive experiments on both synthetic and real datasets.

\section{ACKNOWLEDGEMENTS}

This work is partially sponsored by grants from NSF CyberTrust, NSF NetSE, an IBM SUR grant, and a grant from Intel Research Council. The authors would also like to thank the ACM TODS editors and anonymous reviewers for their valuable constructive comments. 


\section{REFERENCES}

Adam, N. R. And Worthmann, J. C. 1989. Security-control methods for statistical databases: a comparative study. ACM Comput. Surv. 21, 4, 515-556.

Agrawal, D. And Aggarwal, C. C. 2001. On the design and quantification of privacy preserving data mining algorithms. In PODS '01: Proceedings of the twentieth ACM SIGMOD-SIGACTSIGART symposium on Principles of database systems. ACM, New York, NY, USA, 247-255.

Agrawal, R. And SRIKAnt, R. 1994. Fast algorithms for mining association rules in large databases. In VLDB '94: Proceedings of the 20th International Conference on Very Large Data Bases. Morgan Kaufmann Publishers Inc., San Francisco, CA, USA, 487-499.

Agrawal, R. And SRIKant, R. 2000. Privacy-preserving data mining. SIGMOD Rec. 29, 2, 439-450.

Atzori, M., Bonchi, F., Giannotti, F., And Pedreschi, D. 2008. Anonymity preserving pattern discovery. The VLDB Journal 17, 4, 703-727.

Babcock, B., Babu, S., Datar, M., Motwani, R., and Widom, J. 2002. Models and issues in data stream systems. In PODS '02: Proceedings of the twenty-first ACM SIGMOD-SIGACTSIGART symposium on Principles of database systems. ACM, New York, NY, USA, 1-16.

Bu, S., Lakshmanan, L. V. S., NG, R. T., and Ramesh, G. 2007. Preservation of patterns and input-output privacy. In ICDE'07: Proceedings of the 23th IEEE International Conference on Data Mining. IEEE Computer Society, Washington, DC, USA, 696-705.

CAlders, T. 2004. Computational complexity of itemset frequency satisfiability. In PODS '04: Proceedings of the twenty-third ACM SIGMOD-SIGACT-SIGART symposium on Principles of database systems. ACM, New York, NY, USA, 143-154.

Calders, T. And Goethals, B. 2002. Mining all non-derivable frequent itemsets. In PKDD '02: Proceedings of the 6th European Conference on Principles of Data Mining and Knowledge Discovery. Springer-Verlag, London, UK, 74-85.

Chen, K. And Liu, L. 2005. Privacy preserving data classification with rotation perturbation. In ICDM '05: Proceedings of the Fifth IEEE International Conference on Data Mining. IEEE Computer Society, Washington, DC, USA, 589-592.

Chi, Y., Wang, H., Yu, P. S., And Muntz, R. R. 2006. Catch the moment: maintaining closed frequent itemsets over a data stream sliding window. Knowl. Inf. Syst. 10, 3, 265-294.

Chin, F. Y. And Ozsoyoglu, G. 1981. Statistical database design. ACM Trans. Database Syst. 6, 1, 113-139.

Cox, L. 1980. Suppression methodology and statistical disclosure control. Journal of the American Statistical Association 75, 370, 377-385.

Dalenius, T. And Reiss, S. P. 1980. Data-swapping: A technique for disclosure control. J. Statist. Plann. Inference 6, 73-85.

Denning, D. E. 1980. Secure statistical databases with random sample queries. ACM Trans. Database Syst. 5, 3, 291-315.

Dobkin, D., Jones, A. K., And Lipton, R. J. 1979. Secure databases: protection against user influence. ACM Trans. Database Syst. 4, 1, 97-106.

Evfimievski, A., Srikant, R., Agrawal, R., and Gehrke, J. 2002. Privacy preserving mining of association rules. In KDD '02: Proceedings of the eighth ACM SIGKDD international conference on Knowledge discovery and data mining. ACM, New York, NY, USA, 217-228.

Fellegi, I. P. 1972. On the question of statistical confidentiality. Journal of the American Statistical Association 67, 337, 7-18.

Hore, B., Mehrotra, S., And Tsudik, G. 2004. A privacy-preserving index for range queries. In $V L D B$ '04: Proceedings of the Thirtieth international conference on Very large data bases. VLDB Endowment, Toronto, Canada, 720-731.

Huang, Z., Du, W., And Chen, B. 2005. Deriving private information from randomized data. In SIGMOD '05: Proceedings of the 2005 ACM SIGMOD international conference on Management of data. ACM, New York, NY, USA, 37-48.

ACM Transactions on Database Systems, Vol. , No. , 20 
KantarcioĞLu, M., Jin, J., And Clifton, C. 2004. When do data mining results violate privacy? In KDD '04: Proceedings of the tenth ACM SIGKDD international conference on Knowledge discovery and data mining. ACM, New York, NY, USA, 599-604.

Kargupta, H., Datta, S., Wang, Q., And Sivakumar, K. 2003. On the privacy preserving properties of random data perturbation techniques. In ICDM '03: Proceedings of the Third IEEE International Conference on Data Mining. IEEE Computer Society, Washington, DC, USA, 99.

LeFevre, K., DeWitt, D. J., and Ramakrishnan, R. 2006. Mondrian multidimensional kanonymity. In ICDE '06: Proceedings of the 22nd International Conference on Data Engineering. IEEE Computer Society, Washington, DC, USA, 25.

Li, F., Sun, J., Papadimitriou, S., Mihaila, G. A., and Stanoi, I. 2007. Hiding in the crowd: Privacy preservation on evolving streams through correlation tracking. In ICDE'07: Proceedings of the 23th IEEE International Conference on Data Mining. IEEE Computer Society, Washington, DC, USA, 686-695.

Lindell, Y. And PinkAS, B. 2000. Privacy preserving data mining. In CRYPTO '00: Proceedings of the 20th Annual International Cryptology Conference on Advances in Cryptology. SpringerVerlag, London, UK, 36-54.

LukAsiewicz, T. 2001. Probabilistic logic programming with conditional constraints. ACM Trans. Comput. Logic 2, 3, 289-339.

Machanavajuhala, A., Gehrke, J., Kifer, D., and Venkitasubramaniam, M. 2006. l-diversity: Privacy beyond k-anonymity. In ICDE'06: Proceedings of the 22th IEEE International Conference on Data Mining. IEEE Computer Society, Washington, DC, USA, 24.

O'Connor, L. 1993. The inclusion-exclusion principle and its applications to cryptography. Cryptologia 17, 1, 63-79.

PARK, H. AND ShIm, K. 2007. Approximate algorithms for k-anonymity. In SIGMOD '0\%: Proceedings of the 2007 ACM SIGMOD international conference on Management of data. ACM, New York, NY, USA, 67-78.

Shoshani, A. 1982. Statistical databases: Characteristics, problems, and some solutions. In $V L D B$ '82: Proceedings of the 8th International Conference on Very Large Data Bases. Morgan Kaufmann Publishers Inc., San Francisco, CA, USA, 208-222.

Sweeney, L. 2002. k-anonymity: a model for protecting privacy. Int. J. Uncertain. Fuzziness Knowl.-Based Syst. 10, 5, 557-570.

Traub, J. F., Yemini, Y., and Woźniakowski, H. 1984. The statistical security of a statistical database. ACM Trans. Database Syst. 9, 4, 672-679.

VAidya, J. AND Clifton, C. 2002. Privacy preserving association rule mining in vertically partitioned data. In KDD '02: Proceedings of the eighth ACM SIGKDD international conference on Knowledge discovery and data mining. ACM, New York, NY, USA, 639-644.

VAvasis, S. A. 1990. Quadratic programming is in np. Inf. Process. Lett. 36, 2, 73-77.

WAng, K., Fung, B. C. M., AND Yu, P. S. 2007. Handicapping attacker's confidence: an alternative to k-anonymization. Knowl. Inf. Syst. 11, 3, 345-368.

WANG, T. AND LiU, L. 2008. Butterfly: Protecting output privacy in stream mining. In ICDE '08: Proceedings of the 2008 IEEE 24th International Conference on Data Engineering. IEEE Computer Society, Washington, DC, USA, 1170-1179.

Wang, T., Meng, S., Bamba, B., Liu, L., And Pu, C. 2009. A general proximity privacy principle. In ICDE '09: Proceedings of the 2009 IEEE International Conference on Data Engineering. IEEE Computer Society, Washington, DC, USA, 1279-1282. 